\title{
Deficit Irrigation of Durum Wheat (Triticum durum Desf): Effects on Total Dry Matter Production, Light Interception and Radiation Use Efficiency Under Different Nitrogen Rates
}

\author{
Hatem Cheikh M'hamed ${ }^{1}$, Mourad Rezig ${ }^{2} \&$ Mbarek Ben Naceur ${ }^{1}$ \\ ${ }^{1}$ Institut National de la Recherche Agronomique, Tunisie \\ ${ }^{2}$ Institut National de Recherches en Génie Rural Eaux et Forêts, Tunisie \\ Correspondence: Mourad Rezig, Institut National de Recherches en Génie Rural Eaux et Forêts, Rue Hédi \\ Elkarray, Ariana, Tunisie. Tel: 216-98-576-500.E-mail:rezigue_mourad@yahoo.fr
}

\author{
Received: September 9, 2014 Accepted: October 30, 2014 Online Published: November 4, 2014 \\ doi:10.5539/sar.v4n1p26 \\ URL: http://dx.doi.org/10.5539/sar.v4n1p26
}

\begin{abstract}
On-farm trial was conducted from 2005 to 2008 to test the hypothesis that reduction of total dry matter (TDM) in crops can occur after a decreased radiation use efficiency (RUE) due to shortage of nitrogen and irrigation, we applied three irrigations treatments (D1, D2 and D3) and four nitrogen rates (N1, N2, N3 and N4). Photosynthetic active radiation absorbed or cumulative light interception (PARabs) and RUE of Durum wheat were measured. Results showed that D1N1 treatment recorded the highest LAI, PARabs, TDM and RUE. The maximum LAI was obtained 140 DAS (days after sowing) under treatment D1N2 (6.42) and the lowest LAI at the same phase belonged to treatment D2N4 (3.86). At the harvest, the maximum of TDM was $1487 \mathrm{~g} \mathrm{~m}^{-2}$ recorded under treatment D1N1. The minimum value obtained was $930 \mathrm{~g} \mathrm{~m}^{-2}$ under treatment D3N4. Also, PARabs was improved under D1N1 and D1N2 treatments. With reduced $\mathrm{N}$ application rates and irrigation doses, PARabs was decreased and the lowest values were observed under D3N4 condition. The RUE, varied from $1.55 \mathrm{~g} \mathrm{MJ}^{-1}$ (D1N1) to $1.24 \mathrm{~g}$ $\mathrm{MJ}^{-1}$ (D3N4), was affected and decreased under deficit irrigation and low nitrogen conditions. In conclusion, the results of this study seem to show that D1N1 and D1N2 treatments can be beneficial for Durum wheat under field conditions in semi arid zone of Tunisia, for the purpose of improving RUE and maximizing grain yield.
\end{abstract}

Keywords: durum wheat, total dry matter, leaf area index, light interception, radiation use efficiency

\section{Introduction}

Durum wheat (Durum Triticum Desf) is one of the most important staple crops in the semi arid areas of North Africa. Agriculture in this region, especially in Tunisia is primarily based on rainfed cereals integrated with small ruminants production. Consequently, water deficiency and as well as low availability of nutrition (particularly nitrogen $(\mathrm{N})$ ) often limit wheat growth and its production potential (Oweis \& Hachum, 2003). Reports have shown that wheat is sensitive to water and $\mathrm{N}$ at certain physiological growth stages. Nitrogen is the major mineral nutrient for plants and plays a central role in the production of all plant proteins (Sinclair \& Weiss, 2010). In Tunisia, cereal yields are subject to significant fluctuations, given the inter-annual variability of rainfall (Sakiss et al., 1994). The scarcity and uneven distribution of precipitation in this area are a very serious problem especially in recent years, probably due to climate change. As $\mathrm{N}$ fertilizer responses are directly related to rainfall under dryland conditions (Campbell et al., 1993a; Pala et al., 1996), $\mathrm{N}$ use should be correspondingly greater and rational, when supplemental irrigation is applied. However, the response of wheat to irrigation water is dependent on the nitrogen applied (Aggarwal \& Karla, 1994; Oweis et al., 1999). For this purpose, due to the growing water scarcity in Tunisia, as well as to the economic and environmental reasons, today's challenge lies in maximizing production using optimal and scheduled irrigation water doses that saves water (as deficit irrigation) and adequate supply of nitrogen fertilizer essential for the expansion and photosynthetic functioning of plant canopies (Grindlay, 1997). In fact, $\mathrm{N}$ deficiency reduces vegetative and reproductive growth with a final impact on the yield (Tewolde \& Fernandez, 1997). Higher rates of N may shift the balance between vegetative and reproductive growth toward excessive vegetative development, thus delaying crop maturity and reducing final yield (Howard et al., 2001; Hamzei, 2011). Water deficit remarkably decreases the nitrogen translocation ratio derived from soil and adversely affects the contributions of nitrogen in various vegetative organs to grain 
nitrogen (Xu et al., 2006; Hamzei, 2011). Several water-saving methods have been developed (Belder et al., 2004; Bouman et al., 2006), among which, deficit irrigation is one of the best techniques that could improve irrigation water use. Variations in dry matter production in response to $\mathrm{N}$ availability could rise from differences in the amount of cumulative intercepted radiation by the canopy (IPARc, MJ. $\mathrm{m}^{-2}$ ), the radiation use efficiency (RUE, $\mathrm{g} \mathrm{MJ}^{-1}$ ) and the partitioning between different organs. Determination of RUE is an important approach for understanding crop growth and yield production (Sinclair \& Muchow, 1999; Katsura et al., 2007, 2008). Both photosynthetic rates and $\mathrm{N}$ content of leaves affect crop RUE. In this context, Muurinen and Peltonen-Sainio (2006), indicated that RUE of cereals varies seasonally and increases at increasing $\mathrm{N}$ application rates. When crop growth is not limited by other factors, the most appropriate measure for RUE is to fit a linear relationship between cumulative biomass accumulation and radiation interception (Sinclair \& Muchow, 1999). When water stress occurs, the relationships among these parameters change and the crop's ability to capture light reduces (Williams \& Boote, 1995). Under water stress conditions, the fraction of intercepted PAR and leaf area index were often used to evaluate the effects of drought stress on crops (Collino et al., 2001). Results recorded by Hamzei and Soltani (2012) showed that for rapeseed the higher RUE was recorded under moderate deficit irrigation (IR2, $4500 \mathrm{~m}^{3}$ water ha ${ }^{-1}$ ) and optimum $\mathrm{N}$ application $\left(\mathrm{NN}, 12 \mathrm{~g} \mathrm{~m}^{-2}\right.$ ). However, the integrated effect of deficit irrigation and nitrogen applications on the water consumption and yield of wheat requires more detailed studies. Also, no information is available on the interactive effects of nitrogen and irrigation regimes on biomass accumulation and radiation use efficiency for Durum wheat production in Tunisia. Therefore, the general objective of this paper was to investigate the appropriate irrigation regime and $\mathrm{N}$ rate to enhance Durum wheat biomass accumulation and RUE under the semi-arid conditions of Tunisia. This investigation will shed lights on the potential of reducing water and $\mathrm{N}$-fertilizer consumption. Specific objectives of this study were (i) to determine how much increase in the Durum wheat biomass potential could be achieved by application of various $\mathrm{N}$ rates and irrigation regimes, (ii) to identify optimum amounts of nitrogen and water consumptions that contribute to the highest biological yield of (Durum wheat. cv. Karim), and (iii) to compare radiation use efficiency across nitrogen rates and irrigation regimes.

\section{Materials and Methods}

\subsection{Experimental Site}

The experiment was carried out in field at the Private farm 'El Khir' located $30 \mathrm{~km}$ south of Tunis, Tunisia ( $36^{\circ}$ $37^{\prime} \mathrm{N}, 10^{\circ} 08^{\prime} 25^{\prime}$ E), during three successive growing seasons 2005/2006, 2006/2007 and 2007/2008. The climate is semi-arid. The annual rainfall average is about $400 \mathrm{~mm}$. The soil had a clay texture with $180 \mathrm{~mm} \mathrm{~m}^{-1}$ total available water and $1.8 \mathrm{~g} \mathrm{l}^{-1}$ water salinity. The soil bulk density varies from 1.25 to 1.55 from the surface to the depth. The Soil Organic Matter content (SOM \%) are 1.22, 0.9, 0.75 and 0.75 respectively for $0-20 \mathrm{~cm}$, $20-40 \mathrm{~cm}, 40-60 \mathrm{~cm}$ and $60-100 \mathrm{~cm}$ horizons. The $\mathrm{pH}$ of soil varies from 8.1 to 8.5 .

\subsection{Plant Material}

The plant material is composed of one variety of durum wheat "Triticum durum Desf" (Karim). Wheat was sawn at a rate of $180 \mathrm{Kg} \mathrm{ha}^{-1}$ with a drill machine in 2005 on November $24^{\text {th }}$, in 2006 on $31^{\text {th }}$ November and in 2007 on the $17^{\text {th }}$ of November.

\subsection{Experimental Design}

The experiment covered two treatments ( $T_{1}$ : Nitrogen rates and $T_{2}$ : water regimes). $T_{1}$ consisted of four nitrogen rates $\left(\mathrm{N} 1=150 \mathrm{~kg} \mathrm{~N} / \mathrm{ha} ; \mathrm{N} 2=100 \mathrm{~kg} \mathrm{~N} / \mathrm{ha} ; \mathrm{N} 3=50 \mathrm{~kg} \mathrm{~N} / \mathrm{ha}\right.$ and N4=0 kg N/ha). $\mathrm{T}_{2}$ consisted of three water regimes and was monitored (D1 $=$ Full irrigated with $100 \%$ ETM, D2 $=70 \%$ ETM and D3 $=40 \%$ ETM). The experimental design was Split Plot with 3 replications, allowing having 96 elementary plots. The main factor is irrigation regime and the secondary factor is nitrogen rates. Eight meters interval band was maintained between the water regimes treatments and two meters in the case of the nitrogen fertilization elementary plots. The application of all nitrogen rates tested were made $30 \%$ at 6 leafs stage, $40 \%$ at tillering stage and $30 \%$ at stem elongation stage. Treatments descriptions of irrigation regimes are represented in Table 1. 
Table 1. Treatment description

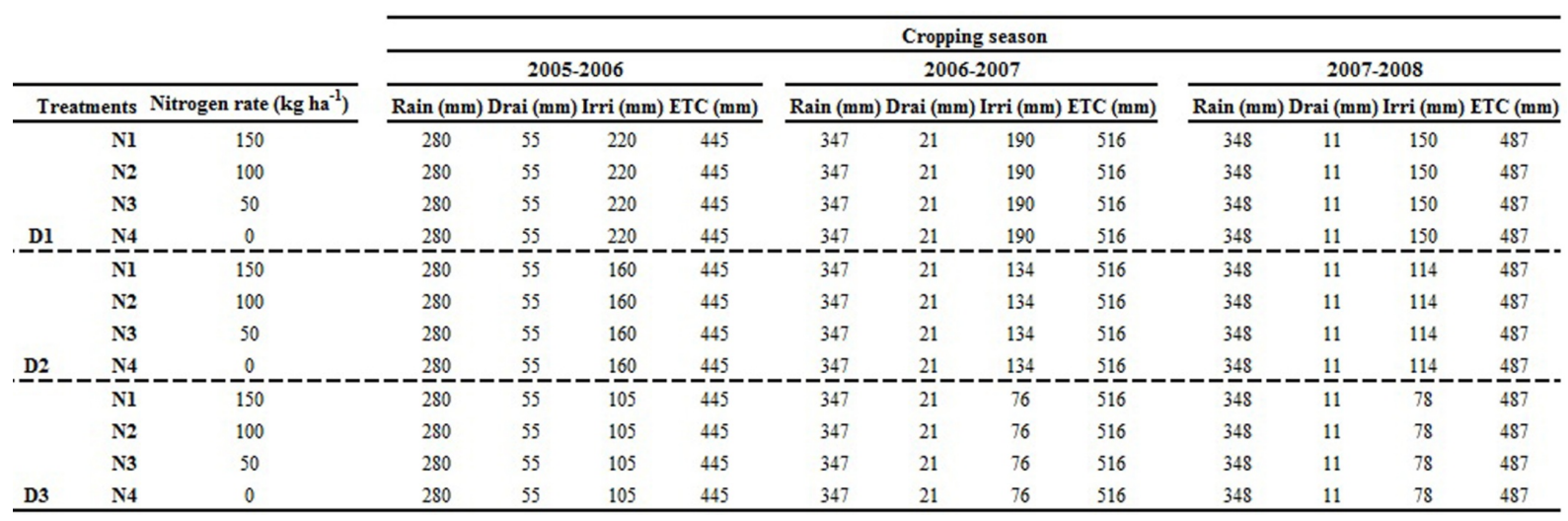

P: Rainfall, Drai: Drainage, Irri: Irrigation, ETC: Crop Evapotranspiration.

\subsection{Field Measurements}

\subsubsection{Climatic Data}

Weather data were recorded daily by automatic agrometeorological station. Collected data were minimum and maximum temperatures (Tmin and Tmax), minimum and maximum air relative humidities (HRmin and HRmax), wind speed (V) and rainfall (P) during the three growing seasons (2005/2006; 2006/2007 and 2007/2008). Reference evapotranspiration (ET0) and solar radiation (Rs, MJ $\mathrm{m}^{-2} \mathrm{~d}^{-1}$ ) were estimated by the MABIA-ET0 software (Jabloun \& Sahli, 2008) using the FAO-Penman-Monteith approach (Allen et al., 1998). The daily Rs were used to calculate the daily photosynthetically active radiation incident (PAR0 $=\mathrm{RS} / 2$ ) (Monteith \& Unsworth, 1990).

\subsubsection{Leaf Area Index, Total Dry Matter Production and Radiation Use Efficiency}

The observations were made on Leaf Area Index (LAI) and total dry matter (TDM g m ${ }^{-2}$ ). In 2005-2006, sampling wheat was collected for growth analysis using one square meter after 45, 70, 99, 118, 138, 164, 204 days of sowing (DAS). In 2006-2007, the sampling was achieved at 45, 67, 92, 114, 134, 164, 198 DAS. In 2007-2008, plants were collected at 45, 83, 104, 124, 140, 160, 211 DAS. At each sampling date, LAI and dry matter weight were measured. The measure of TDM was made using a precision balance (Sartorius, Model PB3001) after oven drying at $65^{\circ} \mathrm{C}$. Leaf area was measured using planimeter type CID Inc-Cl-202.

\subsection{Theoretical Formulations}

\subsubsection{Estimation of the Daily Radiation Interception}

The fraction of intercepted radiation (Fi) was calculated from measurements of LAI using the exponential equation as suggested by Monteith and Elston (1983).

$$
\mathrm{Fi}=1-\mathrm{e}^{\left(-\mathrm{K}^{*} \mathrm{LAI}\right)}
$$

Where $\mathrm{k}$ is the extinction coefficient for total solar radiation. The $\mathrm{k}$ value of 0.45 was used for wheat as described by Jamieson et al. (1995).

Photosynthetically active radiation absorbed by wheat was calculated using the formula of Beer (Manrique et al., 1991):

$$
\text { PARabs }=\text { PAR0 } * \mathrm{Fi}
$$

Where PAR0 is photosynthetically active radiation incident, which is equal to half the solar radiation (Monteith \& Unsworth, 1990).

\subsubsection{Estimation of the Radiation Use Efficiency}

Radiation use efficiency (RUE) of wheat was calculated according the formula below:

$$
\text { RUE }=\frac{\text { TDM }}{\text { PARabs }}
$$




\subsection{Statistical Analysis}

Data collected for all measured parameters were subjected to tests of variance analysis, using Statistical Analysis System software (SAS, 1985). This variance analysis was completed by "multiple comparisons of means" with Newman Keuls test. LSD (Least Significant Difference) was used for comparing treatment group means at 0.05\% (Little \& Hill, 1978).

\section{Results}

\subsection{Leaf Area Index}

Figure 1 shows the kinetics of Leaf area index LAI for the three wheat growing season at the various nitrogen rates (N1, N2, N3 and N4) and under the three irrigations rates (D1, D2 and D3).

As shown in Table 2, the differences between treatments (N1, N2, N3 and N4) under the three irrigation volumes (D1, D2 and D3) were significant $(\mathrm{P}<0.05)$ at maximum growth.

In the 2005-2006 growing season, the highest LAI (4.91) was obtained 138 DAS from treatment D1N1 and the lowest LAI (3.86) at the same phase belonged to treatment D2N4. In the 2006-2007 growing season, the highest (5.78) and the lowest LAI (4.9) were obtained 134 DAS from treatment D1N1 and D2 N4 respectively. In the 2007-2008 growing season, the maximum (6.42) and minimum LAI (5.02) were achieved 140 DAS from D1N2 and D2N4 treatment respectively. The effect of irrigation on LAI was yearly depending. So in the first and the second experiment respectively at 138 and 134 DAS, ANOVAANOVA analysis shows that there is no significant effect $(\mathrm{P}>0.05)$ of irrigation treatments on LAI. Nevertheless for the third experimentation 2007-2008, there was significant effect $(\mathrm{P}<0.05)$ of irrigation treatment on LAI (Table 2). For the three experiments, ANOVA analysis shows that nitrogen application significantly $(\mathrm{P}<0.001)$ increased the LAI. The maximum values of wheat Leaf area index (LAI max) were achieved for treatment (N1 and N2) and the lowest for treatment N4. The combined effect of irrigation regime and nitrogen application has a significant effect $(\mathrm{P}<0.01)$ on LAI. So, for the three experiments (2006, 2007 and 2008), the maximum value of Leaf area index (LAI max) was recorded in treatment D1N1 for the first and the second experiment (4.91 and 5.78 respectively) and in D1N2 for the third experiment (6.42). The lowest LAI was respectively equivalent to $(3.86 ; 4.9$ and 5.02$)$ in treatment D2N4. 

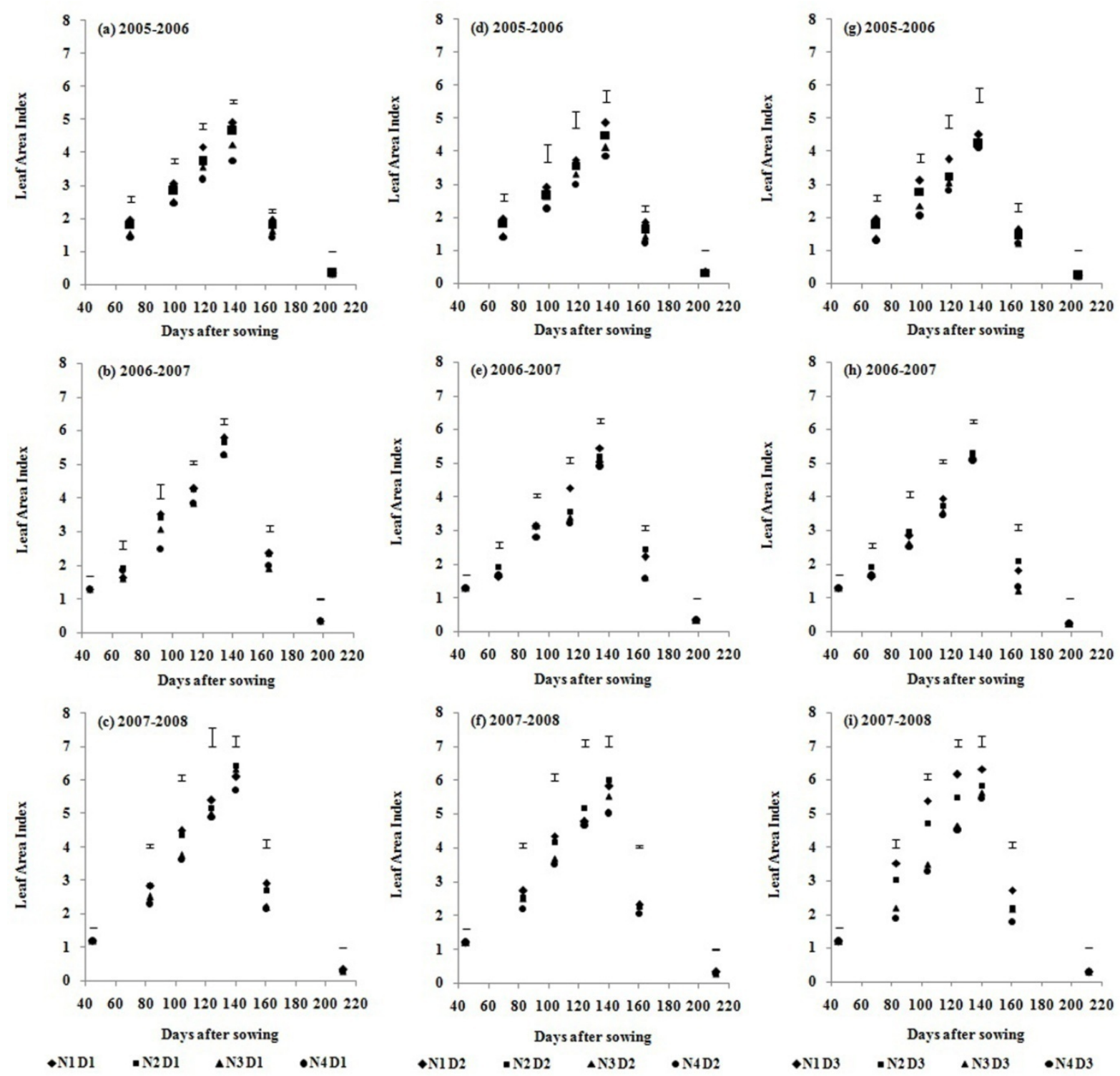

Figure 1. Leaf area index of wheat during the three experiments at the four nitrogen rates (N1, N2, N3 and N4) and under different water doses: D1 (a, b and c); D2 (d, e and f) and D3 (g, h and i). The vertical bars represent the least significant difference at $5 \%$ (LSD) 
Table 2. Leaf area index of wheat at the four nitrogen rates (N1, N2, N3 and N4) and under three irrigation amounts (D1, D2 and D3) during the three campaigns (2006, 2007 and 2008)

\begin{tabular}{|c|c|c|c|c|c|c|c|c|c|c|c|c|c|c|c|c|c|c|c|c|c|}
\hline \multirow[b]{2}{*}{ DAS } & \multicolumn{7}{|c|}{$2005-2006$} & \multicolumn{7}{|c|}{ 2006-2007 } & \multicolumn{7}{|c|}{$2007-2008$} \\
\hline & 45 & 70 & 99 & 118 & 138 & 164 & 204 & 45 & 67 & 92 & 114 & 134 & 164 & 198 & 45 & 83 & 104 & 124 & 140 & 160 & 211 \\
\hline DIN1 & $1.31 \mathrm{a}$ & $1.94 \mathrm{a}$ & $3.06 \mathrm{ab}$ & $4.17 \mathrm{a}$ & $4.91 \mathrm{a}$ & $1.94 \mathrm{a}$ & $0.3 \mathrm{a}$ & $1.3 \mathrm{a}$ & $1.62 \mathrm{c}$ & $3.53 \mathrm{a}$ & $4.27 \mathrm{a}$ & $5.78 \mathrm{a}$ & $2.37 \mathrm{ab}$ & $0.3 \mathrm{a}$ & $1.2 \mathrm{a}$ & $2.85 \mathrm{c}$ & $4.5 \mathrm{c}$ & $5.4 \mathrm{~b}$ & $6.12 \mathrm{bc}$ & $2.92 \mathrm{a}$ & $0.3 \mathrm{a}$ \\
\hline DIN2 & $1.31 \mathrm{a}$ & $1.82 \mathrm{ab}$ & $2.83 \mathrm{~cd}$ & $3.73 \mathrm{~b}$ & $4.65 \mathrm{ab}$ & $1.82 \mathrm{a}$ & $0.3 \mathrm{a}$ & $1.3 \mathbf{a}$ & $1.91 \mathrm{a}$ & $3.41 \mathrm{a}$ & $4.26 \mathrm{a}$ & $5.64 \mathrm{~b}$ & $2.33 \mathrm{ab}$ & $0.3 \mathrm{a}$ & $1.2 \mathrm{a}$ & $2.85 \mathrm{a}$ & $4.37 \mathrm{c}$ & $5.16 \mathrm{bc}$ & $6.42 \mathrm{a}$ & $2.72 \mathrm{~b}$ & $0.3 \mathrm{a}$ \\
\hline DIN3 & $1.31 \mathrm{a}$ & $1.68 \mathrm{bc}$ & $2.52 \mathrm{efg}$ & $3.57 \mathrm{bc}$ & $4.23 \mathrm{c}$ & $1.62 \mathrm{~b}$ & $0.3 \mathrm{a}$ & $1.3 \mathrm{a}$ & $1.62 \mathrm{c}$ & $3.06 \mathrm{bc}$ & $3.83 \mathrm{bc}$ & $5.31 \mathrm{~d}$ & $1.9 \mathrm{e}$ & $0.3 \mathrm{a}$ & $1.2 \mathrm{a}$ & $2.51 \mathrm{~d}$ & $3.75 \mathrm{e}$ & $4.98 \mathrm{~cd}$ & $6.34 \mathrm{ab}$ & $2.02 \mathrm{e}$ & $0.3 \mathbf{a}$ \\
\hline DIN4 & $1.31 \mathrm{a}$ & $1.6 \mathrm{c}$ & $2.47 \mathrm{fg}$ & $3.19 \mathrm{de}$ & $3.74 \mathrm{e}$ & $1.42 \mathrm{c}$ & $0.3 \mathrm{a}$ & $1.3 \mathrm{a}$ & $1.85 \mathrm{ab}$ & $2.47 \mathrm{f}$ & $3.83 \mathrm{bc}$ & $5.28 \mathrm{de}$ & $1.99 \mathrm{de}$ & $0.3 \mathrm{a}$ & $1.2 \mathrm{a}$ & $2.3 \mathrm{e}$ & $3.62 \mathrm{ef}$ & $49 \mathrm{~cd}$ & $5.7 \mathrm{ef}$ & 2.16 cde & $0.3 \mathrm{a}$ \\
\hline D2N1 & $1.31 \mathrm{a}$ & $1.95 \mathrm{a}$ & $2.92 \mathrm{abc}$ & $3.75 \mathrm{~b}$ & $4.86 \mathrm{a}$ & $1.82 \mathrm{a}$ & $0.3 \mathrm{a}$ & $1.3 \mathrm{a}$ & $1.64 \mathrm{c}$ & $3.12 \mathrm{~b}$ & $4.26 \mathrm{a}$ & $5.45 \mathrm{c}$ & $2.22 \mathrm{bc}$ & $0.3 \mathbf{a}$ & $1.2 \mathrm{a}$ & $2.73 \mathrm{c}$ & $4.35 \mathrm{~cd}$ & $4.8 \mathrm{cde}$ & $5.82 \mathrm{de}$ & $2.32 \mathrm{c}$ & $0.3 \mathrm{a}$ \\
\hline D2N2 & $1.31 \mathrm{a}$ & $1.82 \mathrm{ab}$ & 2.66 cdef & $3.57 b c$ & $4.5 \mathrm{~b}$ & $1.62 \mathrm{~b}$ & $0.3 \mathrm{a}$ & $1.3 \mathrm{a}$ & $1.91 \mathrm{a}$ & $3.12 \mathrm{~b}$ & $3.54 \mathrm{~d}$ & $5.19 \mathrm{ef}$ & $2.43 \mathrm{a}$ & $0.3 \mathrm{a}$ & $1.2 \mathrm{a}$ & $2.53 \mathrm{~d}$ & $4.17 \mathrm{~d}$ & $6.16 \mathrm{a}$ & $6.02 \mathrm{~cd}$ & $2.25 \mathrm{c}$ & $0.25 \mathrm{~b}$ \\
\hline D2N3 & $1.31 \mathrm{a}$ & $1.68 \mathrm{bc}$ & $2.64 \mathrm{def}$ & $3.32 \mathrm{~cd}$ & $4.12 \mathrm{~cd}$ & $1.42 \mathrm{c}$ & $0.3 \mathrm{a}$ & $1.3 \mathrm{a}$ & $1.72 \mathrm{bc}$ & $3.13 \mathrm{~b}$ & $3.38 \mathrm{e}$ & $5.11 \mathrm{fg}$ & $1.61 \mathrm{f}$ & $0.3 \mathrm{a}$ & $1.2 \mathrm{a}$ & $2.51 \mathrm{~d}$ & $3.69 \mathrm{e}$ & 4.83 cde & $5.53 \mathrm{fg}$ & $2.3 \mathrm{c}$ & $0.25 \mathrm{~b}$ \\
\hline D2N4 & $1.31 \mathrm{a}$ & $1.6 \mathrm{c}$ & $2.26 \mathrm{gh}$ & 2.99 ef & $3.86 \mathrm{de}$ & $1.23 \mathrm{~d}$ & $0.3 \mathrm{a}$ & $1.3 \mathrm{a}$ & $1.65 \mathrm{c}$ & $2.79 \mathrm{de}$ & $3.2 \mathrm{f}$ & $4.9 \mathrm{~h}$ & $1.58 \mathrm{f}$ & $0.3 \mathrm{a}$ & $1.2 \mathrm{a}$ & $2.18 \mathrm{e}$ & $3.5 \mathrm{f}$ & $4.65 \mathrm{de}$ & $5.02 \mathrm{~h}$ & $2.06 \mathrm{de}$ & $0.25 \mathrm{~b}$ \\
\hline D3N1 & $1.31 \mathrm{a}$ & $1.94 \mathrm{a}$ & $3.12 \mathrm{a}$ & $3.76 \mathrm{~b}$ & $4.52 \mathrm{~b}$ & $1.62 \mathrm{~b}$ & $0.2 \mathrm{~b}$ & $1.3 \mathrm{a}$ & $1.64 \mathrm{c}$ & $2.86 \mathrm{~cd}$ & $3.95 \mathrm{~b}$ & $5.1 \mathrm{fg}$ & $1.82 \mathrm{e}$ & $0.2 \mathrm{~b}$ & $1.2 \mathrm{a}$ & $3.53 \mathrm{a}$ & $5.37 \mathrm{a}$ & $6.16 \mathrm{a}$ & $6.32 \mathrm{ab}$ & $2.72 \mathrm{~b}$ & $0.25 \mathrm{~b}$ \\
\hline D3N2 & $1.31 \mathrm{a}$ & $1.77 \mathrm{~b}$ & $2.78 \mathrm{cde}$ & $3.25 \mathrm{de}$ & $4.23 \mathrm{c}$ & $1.45 \mathrm{c}$ & $0.2 \mathrm{~b}$ & $1.3 \mathrm{a}$ & $1.91 \mathrm{a}$ & $2.97 \mathrm{bcd}$ & $3.74 \mathrm{c}$ & $5.29 \mathrm{de}$ & $2.1 \mathrm{~cd}$ & $0.2 \mathrm{~b}$ & $1.2 \mathrm{a}$ & $3.01 \mathrm{~b}$ & $4.69 \mathrm{a}$ & $5.48 \mathrm{~b}$ & $5.83 \mathrm{de}$ & $2.2 \mathrm{~cd}$ & $0.25 \mathrm{~b}$ \\
\hline D3N3 & $1.31 \mathrm{a}$ & $1.68 \mathrm{bc}$ & $2.36 \mathrm{~g}$ & $3.05 \mathrm{def}$ & $4.21 \mathrm{c}$ & $1.22 \mathrm{~d}$ & $0.2 \mathrm{~b}$ & $1.3 \mathrm{a}$ & $1.72 \mathrm{bc}$ & $2.62 \mathrm{ef}$ & $3.57 \mathrm{~d}$ & $5.22 \mathrm{de}$ & $1.2 \mathrm{~g}$ & $0.2 \mathrm{~b}$ & $1.2 \mathrm{a}$ & $2.18 \mathrm{e}$ & $3.49 \mathrm{f}$ & $4.64 \mathrm{de}$ & $5.62 \mathrm{efg}$ & $2.6 \mathrm{~b}$ & $0.25 \mathrm{~b}$ \\
\hline D3N4 4 & $1.31 \mathrm{a}$ & $1.6 \mathrm{c}$ & $2.05 \mathrm{~h}$ & $2.81 \mathrm{f}$ & $4.12 \mathrm{~cd}$ & $1.22 \mathrm{~d}$ & $0.2 \mathrm{~b}$ & $1.3 \mathrm{a}$ & $1.65 \mathrm{c}$ & $2.52 \mathrm{f}$ & $3.46 \mathrm{de}$ & $5.08 \mathrm{~g}$ & $1.33 \mathrm{~g}$ & $0.2 \mathrm{~b}$ & $1.2 \mathrm{a}$ & $1.88 \mathrm{f}$ & $3.27 \mathrm{~g}$ & $4.5 \mathrm{e}$ & $5.43 \mathrm{~g}$ & $1.78 \mathrm{f}$ & $0.25 b$ \\
\hline $\operatorname{LSD}(5 \%)$ & 0 & 0.169 & 0.269 & 0.319 & 0.265 & 0.155 & 0.083 & 0 & 0.168 & 0.212 & 0.14 & 0.101 & 0.185 & 0.028 & 0 & 0.147 & 0.187 & 0.385 & 0.268 & 0.163 & 0.03 \\
\hline & $1 \mathrm{NS}$ & $0.9275 \mathrm{NS}$ & $0.1288 \mathrm{NS}$ & $0.002 * *$ & $0.2701 \mathrm{NS}$ & $.0001^{* * *}$ & $0.0001^{* * *}$ & $\overline{0.0001 * * *}$ & $0.9275 \mathrm{NS}$ & $0.1288 \mathrm{NS}$ & $0.0002=$ & $0.2701 \mathrm{NS}$ & $0.0001^{* * *}$ & $0.0001^{* * *}$ & $0.0001^{* * *}$ & $0.9275 \mathrm{NS}$ & $0.1288 \mathrm{~N}$ & $0.0002 * *$ & .0001 & $0.0001 * *$ & $0.0001^{*}$ \\
\hline $\mathrm{N}$ & $1 \mathrm{NS}$ & $0.0001 * \cdots$ & $0.0001 * \cdots$ & $0.0001 * *$ & $0.0001 * \cdots$ & $.0001 \cdots *$ & 1 NS & 1 NS & $0.0001 * \cdots$ & $0.0001^{* * *}$ & $0.0001^{* *}$ & $0.0001 * * *$ & $0.0001 * * *$ & $1 \mathrm{NS}$ & $1 \mathrm{NS}$ & $0.0001 * * *$ & $0.0001^{*}$ & $0.0001 *$ & $.0001 * \cdots$ & $0.0001 * * *$ & $1 \mathrm{NS}$ \\
\hline$D^{*} \mathrm{~N}$ & $0.0014^{*}$ & $9989 \mathrm{NS}$ & $0.0942 \mathrm{NS}$ & $0.8332 \mathrm{NS}$ & $0.006 s^{* *}$ & $4413 \mathrm{NS}$ & $0.0001^{* * *}$ & $0.0014^{* *}$ & $0.9989 \mathrm{NS}$ & $0.0942 \mathrm{NS}$ & $0.8332 \mathrm{NS}$ & $0.0068^{* *}$ & $0.4413 \mathrm{NS}$ & $0.0001^{* * *}$ & $0.0014^{* *}$ & $0.9989 \mathrm{NS}$ & $0.0942 \mathrm{NS}$ & $0.8332 \mathrm{NS}$ & $0.0068^{* *}$ & $0.4413 \mathrm{NS}$ & $0.0001^{* *}$ \\
\hline
\end{tabular}

DAS: Days After Sowings, LSD: Least Significant Difference, ${ }^{*}$ significant difference at $5 \%,{ }^{* *}$ significant difference at $0.01 \%, * * *$ significant difference at $0.001 \%$, NS no significant difference at $5 \%$.

\subsection{Total Dry Matter Production}

The Total dry matter accumulation of wheat (TDM) at the various nitrogen rates (N1, N2, N3 and N4) and under the three irrigations rates (D1, D2 and D3) was shown in Figure 2.

Total Dry Matter accumulation (TDM) presented a high variability according to the treatment and year (Figure 2 and Table 3). The maximum of TDM $\left(1487 \mathrm{~g} \mathrm{~m}^{-2}\right)$ was recorded under treatment D1N1 in year 2. The minimum $\left(930 \mathrm{~g} \mathrm{~m}^{-2}\right)$ was reached only in year 3 under treatment $\mathrm{D} 3 \mathrm{~N} 4$. The TDM was significantly $(\mathrm{P}<0.001)$ affected by irrigation doses (D1, D2 and D3) in 2006, 2007 and 2008 growing seasons (Table 3). At harvesting, D1 increased the TDM in the treatment N1 compared to D2 and D3, respectively (from 2.9 to $5.8 \%$ ) and (from 11.7 to $15.1 \%$ ). Similarly, the treatment D1N2 has increased TDM compared to D2N2 and D3N2, respectively from 1.7 to $6.1 \%$ and from 6.1 to $15.9 \%$. Likewise for the treatment D1N4 an increase in TDM was registered from 4.9 to $9.3 \%$ and from 6.9 to $13.2 \%$ next to in $\mathrm{D} 2 \mathrm{~N} 4$ and $\mathrm{D} 3 \mathrm{~N} 4$ respectively. ANOVA revealed that TDM was significantly (P< 0.001 ) influenced by nitrogen rates. Over all years and irrigation regimes, the wheat TDM was the greatest in the two treatments N1 and N2 and the least in the treatment N4 [Figure 2 and Table 3]. Treatments that not received nitrogen fertilizer produced the smallest TDM. Therefore, the application of nitrogen in N1 and N2 significantly ( $\mathrm{P}$ $<0.001$ ) increased TDM compared to N3 and N4. Indeed, in the irrigation regime D1, the treatment N1 improved the TDM compared to N3 and N4 rates, respectively from 11.7 to $12.6 \%$ and from 15 to $22.3 \%$. Also, in the second regime D2, the treatment $\mathrm{N} 1$ has enhanced TDM compared to N3 and N4, respectively from 9.9 to $12.3 \%$ and from 17.1 to $24.4 \%$. Similarly, in the third regime D3, the TDM in N1 was higher than N3 and N4, respectively from 7.3 to $12 \%$ and from 12.8 to $22.7 \%$. The combined effect of irrigation regime and nitrogen application had a significant effect $(\mathrm{P}<0.05)$ on the TDM production only during the second and third experiments (Table 3$)$. So, at harvest, the D1N1 has improved TDM compared to D4N4 about 23\%, 26.2\% and 31.7\%, respectively for the three experiments 2006, 2007 and 2008.

Table 3. Total Dry Matter accumulation of wheat at the four nitrogen rates (N1, N2, N3 and N4) and under three irrigation doses (D1, D2 and D3) during the three campaigns (2006, 2007 and 2008)

\begin{tabular}{|c|c|c|c|c|c|c|c|c|c|c|c|c|c|c|c|c|c|c|c|c|c|}
\hline \multirow[b]{2}{*}{ DAS } & \multicolumn{7}{|c|}{$2005-2006$} & \multicolumn{7}{|c|}{$2006-2007$} & \multicolumn{7}{|c|}{$2007-2008$} \\
\hline & 45 & 70 & 99 & 118 & 138 & 164 & 204 & 45 & 67 & 92 & 114 & 134 & 164 & 198 & 45 & 83 & 104 & 124 & 140 & 160 & 211 \\
\hline DIN1 & $85 a$ & $190 \mathrm{a}$ & $380 \mathrm{a}$ & $590 \mathrm{a}$ & $730 \mathrm{a}$ & $1000 \mathrm{a}$ & $1254.66 \mathrm{a}$ & $85 a$ & $135.66 \mathrm{~b}$ & $410.66 \mathrm{a}$ & $610 \mathrm{a}$ & $840.33 \mathrm{a}$ & $1249 \mathrm{a}$ & $1487 \mathrm{a}$ & $80 \mathrm{a}$ & $275 \mathrm{a}$ & $620 \mathrm{a}$ & $800 \mathrm{a}$ & $950 \mathrm{abc}$ & 1150 ab & $1362.33 \mathrm{a}$ \\
\hline DIN2 & 85 a & $165 \mathrm{~b}$ & $340 \mathrm{bc}$ & $540 \mathrm{~b}$ & $700 \mathrm{ab}$ & $950 \mathrm{ab}$ & $1222.66 \mathrm{ab}$ & $85 a$ & $153.66 \mathrm{a}$ & $400.66 \mathrm{a}$ & $600.66 \mathrm{a}$ & $820.33 \mathrm{ab}$ & $1200.66 \mathrm{~b}$ & $1454.33 \mathrm{ab}$ & $80 \mathrm{a}$ & $270 \mathrm{a}$ & $601 \mathrm{ab}$ & $770 \mathrm{ab}$ & $990 \mathrm{a}$ & $1180 \mathrm{a}$ & $1331 \mathrm{a}$ \\
\hline DIN3 & $85 a$ & $130 \mathrm{~d}$ & 280 ef & $500 \mathrm{c}$ & $620 \mathrm{~cd}$ & $850 \mathrm{~cd}$ & $1100.33 \mathrm{de}$ & 85 a & $135.33 \mathrm{~b}$ & $375.66 \mathrm{~b}$ & $550.33 \mathrm{~b}$ & $791 \mathrm{~cd}$ & $1080.66 \mathrm{de}$ & $1300.33 \mathrm{~d}$ & $80 \mathrm{a}$ & $250.66 \mathrm{ab}$ & $550.33 \mathrm{~cd}$ & $730 \mathrm{bcd}$ & $970 \mathrm{ab}$ & $1100 \mathrm{bcd}$ & $1203.33 \mathrm{bc}$ \\
\hline DIN4 & 85 a & $125 \mathrm{e}$ & $270 \mathrm{f}$ & $420 \mathrm{e}$ & $0 \mathrm{f}$ & $800 \mathrm{de}$ & $1038.66 \mathrm{fg}$ & 85 a & $127.66 \mathrm{c}$ & $275 \mathrm{e}$ & $550.33 \mathrm{~b}$ & $781 \mathrm{cde}$ & $1100.33 \mathrm{~cd}$ & $1264.33 \mathrm{de}$ & $80 \mathrm{a}$ & $235.66 \mathrm{~b}$ & $531.66 \mathrm{de}$ & $710 \mathrm{~cd}$ & $880.33 \mathrm{de}$ & $1025.33 \mathrm{ef}$ & $1058.66 \mathrm{e}$ \\
\hline D2N1 & $85 a$ & $190 \mathrm{a}$ & $365 \mathrm{ab}$ & $550 \mathrm{~b}$ & $710 \mathrm{ab}$ & $950 \mathrm{ab}$ & $1191.33 \mathrm{bc}$ & $85 \mathrm{a}$ & $135.66 \mathrm{~b}$ & $326 \mathrm{c}$ & $600 \mathrm{a}$ & $800.66 \mathrm{bc}$ & $1130.66 \mathrm{c}$ & $1401.33 \mathrm{c}$ & $80 \mathrm{a}$ & $265 \mathrm{a}$ & $600 \mathrm{ab}$ & $780 \mathrm{ab}$ & $920 \mathrm{~cd}$ & $1140 \mathrm{ab}$ & $1322 \mathrm{a}$ \\
\hline D2N2 & 85 a & $165 \mathrm{~b}$ & $320 \mathrm{dc}$ & $510 \mathrm{c}$ & $680 \mathrm{ab}$ & $900 \mathrm{bc}$ & $1148.33 \mathrm{~cd}$ & $85 \mathrm{a}$ & $153.66 \mathrm{a}$ & $325.66 \mathrm{c}$ & $480.33 \mathrm{~cd}$ & $770.33 \mathrm{def}$ & $1225.66 \mathrm{ab}$ & $1430.66 \mathrm{bc}$ & $80 \mathrm{a}$ & $250 \mathrm{ab}$ & $580.66 \mathrm{bc}$ & 750 abc & $950 \mathrm{abc}$ & $1120 \mathrm{bc}$ & $1250 \mathrm{~b}$ \\
\hline D2N3 & 85 a & $130 \mathrm{~d}$ & 0 def & $470 \mathrm{~d}$ & de & $800 \mathrm{de}$ & $1048.33 \mathrm{ef}$ & 85 a & $135.33 \mathrm{~b}$ & $325.33 \mathrm{c}$ & $460.66 \mathrm{~d}$ & $750.66 \mathrm{fg}$ & 1040.66 ef & $1262 \mathrm{de}$ & $80 \mathrm{a}$ & $235.66 \mathrm{~b}$ & $530.33 \mathrm{de}$ & $700 \mathrm{~cd}$ & $940 \mathrm{bc}$ & 1080 cde & $1160.33 \mathrm{~cd}$ \\
\hline D2N4 & 85 a & $125 \mathrm{e}$ & $230 \mathrm{gh}$ & $400 \mathrm{f}$ & $575 \mathrm{de}$ & $750 \mathrm{e}$ & $987.66 \mathrm{ghi}$ & 85 a & $127.66 \mathrm{c}$ & $270.66 \mathrm{e}$ & $430.33 \mathrm{e}$ & $720.33 \mathrm{~h}$ & $1000.66 \mathrm{f}$ & $1147.33 \mathrm{f}$ & $80 \mathrm{a}$ & $225 \mathrm{bc}$ & $512 \mathrm{e}$ & $680 \mathrm{de}$ & $830.33 \mathrm{f}$ & $975.33 \mathrm{fg}$ & $1000.66 \mathrm{f}$ \\
\hline D3N1 & 85 a & $190 \mathrm{a}$ & $380 \mathrm{a}$ & $550 \mathrm{~b}$ & $670 \mathrm{bc}$ & $900 \mathrm{bc}$ & $1108.33 \mathrm{~d}$ & $85 \mathrm{a}$ & $135.66 \mathrm{~b}$ & $275.66 \mathrm{e}$ & $570.33 \mathrm{~b}$ & 760.66 ef & $1080.33 \mathrm{de}$ & $1262.33 \mathrm{de}$ & $80 \mathrm{a}$ & $250 \mathrm{ab}$ & $580 \mathrm{bc}$ & $750 \mathrm{abc}$ & $850 \mathrm{ef}$ & $1050 \mathrm{de}$ & $1203.33 \mathrm{bc}$ \\
\hline D3N2 & 85 a & $145 \mathrm{c}$ & $305 \mathrm{de}$ & $480 \mathrm{~d}$ & & $835 \mathrm{~cd}$ & $1027.66 \mathrm{fgh}$ & 85 a & $153.66 \mathrm{a}$ & $300.66 \mathrm{~d}$ & $550.66 \mathrm{~b}$ & $779.33 \mathrm{cde}$ & e $1100.66 \mathrm{~cd}$ & $1246.33 \mathrm{e}$ & $80 \mathrm{a}$ & $250.66 \mathrm{ab}$ & $550.33 \mathrm{~cd}$ & $730 \mathrm{bcd}$ & $880 \mathrm{de}$ & $1080 \mathrm{cde}$ & $1250 \mathrm{~b}$ \\
\hline D3N3 & $85 a$ & $130 \mathrm{~d}$ & $260 \mathrm{fg}$ & 410 ef & 570 def & $745 \mathrm{e}$ & $975.66 \mathrm{hi}$ & $85 \mathrm{a}$ & $135.33 \mathrm{~b}$ & $265.33 \mathrm{e}$ & $500 \mathrm{c}$ & $750.33 \mathrm{fg}$ & $920.66 \mathrm{~g}$ & $1170.33 \mathrm{f}$ & $80 \mathrm{a}$ & $225 \mathrm{bc}$ & $512 \mathrm{e}$ & $680 \mathrm{de}$ & $820 \mathrm{fg}$ & $975.33 \mathrm{fg}$ & $1110.66 \mathrm{de}$ \\
\hline D3N4 & $85 a$ & $125 \mathrm{e}$ & $210 \mathrm{~h}$ & $370 \mathrm{~g}$ & $560 \mathrm{ef}$ & $740 \mathrm{e}$ & $966.66 \mathrm{i}$ & $85 a$ & $127.66 \mathrm{c}$ & $255 \mathrm{e}$ & $480.33 \mathrm{~cd}$ & $722.33 \mathrm{gh}$ & $900.66 \mathrm{~g}$ & $1097.33 \mathrm{~g}$ & $80 \mathrm{a}$ & $205 \mathrm{c}$ & $460.66 \mathrm{f}$ & $630 \mathrm{e}$ & $800.33 \mathrm{~g}$ & $925 \mathrm{~g}$ & $930 \mathrm{~g}$ \\
\hline LSD (5\%) & 0 & 4.78 & 31.75 & 14.36 & 53.77 & 68 & 53 & 0 & 3.64 & 21.15 & 22.61 & 28.49 & 46.68 & 39.34 & 0 & 27.29 & 36.44 & 54.03 & 47.44 & 59.6 & 52.6 \\
\hline D & $1 \mathrm{NS}$ & $0.0011^{* *}$ & $0.0043^{* *}$ & $0.0001^{* * *}$ & $0.0068^{* *}$ & $0.0002^{* * *}$ & $0.0001^{* * *}$ & $1 \mathrm{NS}$ & $1 \mathrm{NS}$ & $0.0001^{* * *}$ & $0.0001^{* * *}$ & $0.0001^{* * *}$ & $0.0001^{* * *}$ & $0.0001^{* * *}$ & $1 \mathrm{NS}$ & $0.0024^{* *}$ & $0.0002^{* * *}$ & $0.0035 * *$ & $0.0001^{* *}$ & $0.0001^{* *}$ & $0.0001^{* * *}$ \\
\hline $\mathrm{N}$ & $1 \mathrm{NS}$ & $0.0001^{* * *}$ & $0.0001^{* \cdots *}$ & $0.0001^{* * *}$ & $0.0001^{* * *}$ & $0.0001^{* * *}$ & $0.0001^{* * *}$ & 1 NS 0 & $0.0001 * \cdots$ & $0.0001 * * *$ & $0.0001^{* * *}$ & $0.0001^{* * *}$ & $0.0001^{* * *}$ & $0.0001^{* * *}$ & $1 \mathrm{NS}$ & $0.0001^{* * *}$ & $0.0001^{* * *}$ & $0.0001^{* * *}$ & $0.0001^{* * *}$ & $0.0001^{* * *}$ & $0.0001^{* * *}$ \\
\hline$D^{*} \mathrm{~N}$ & $1 \mathrm{NS}$ & $0.0001^{* * *}$ & $0.0955 \mathrm{NS}$ & $0.0003^{* * *}$ & $0.3988 \mathrm{NS}$ & $0.9189 \mathrm{NS}$ & $0.1327 \mathrm{NS}$ & $1 \mathrm{NS}$ & $1 \mathrm{NS}$ & $0.0001 * \cdots$ & $0.0001^{* \cdots *}$ & $0.1961 \mathrm{NS}$ & $0.0032^{* *}$ & $0.0008^{* * *}$ & $1 \mathrm{NS}$ & $0.9433 \mathrm{NS}$ & $0.8378 \mathrm{NS}$ & $0.961 \mathrm{NS}$ & $0.3184 \mathrm{NS}$ & $0.7291 \mathrm{NS}$ & $0.023^{*}$ \\
\hline
\end{tabular}

DAS: Days After Sowings, LSD: Least Significant Difference, * significant difference at $5 \%$, ** significant difference at $0.01 \%, * * *$ significant difference at $0.001 \%$, NS no significant difference at $5 \%$. 

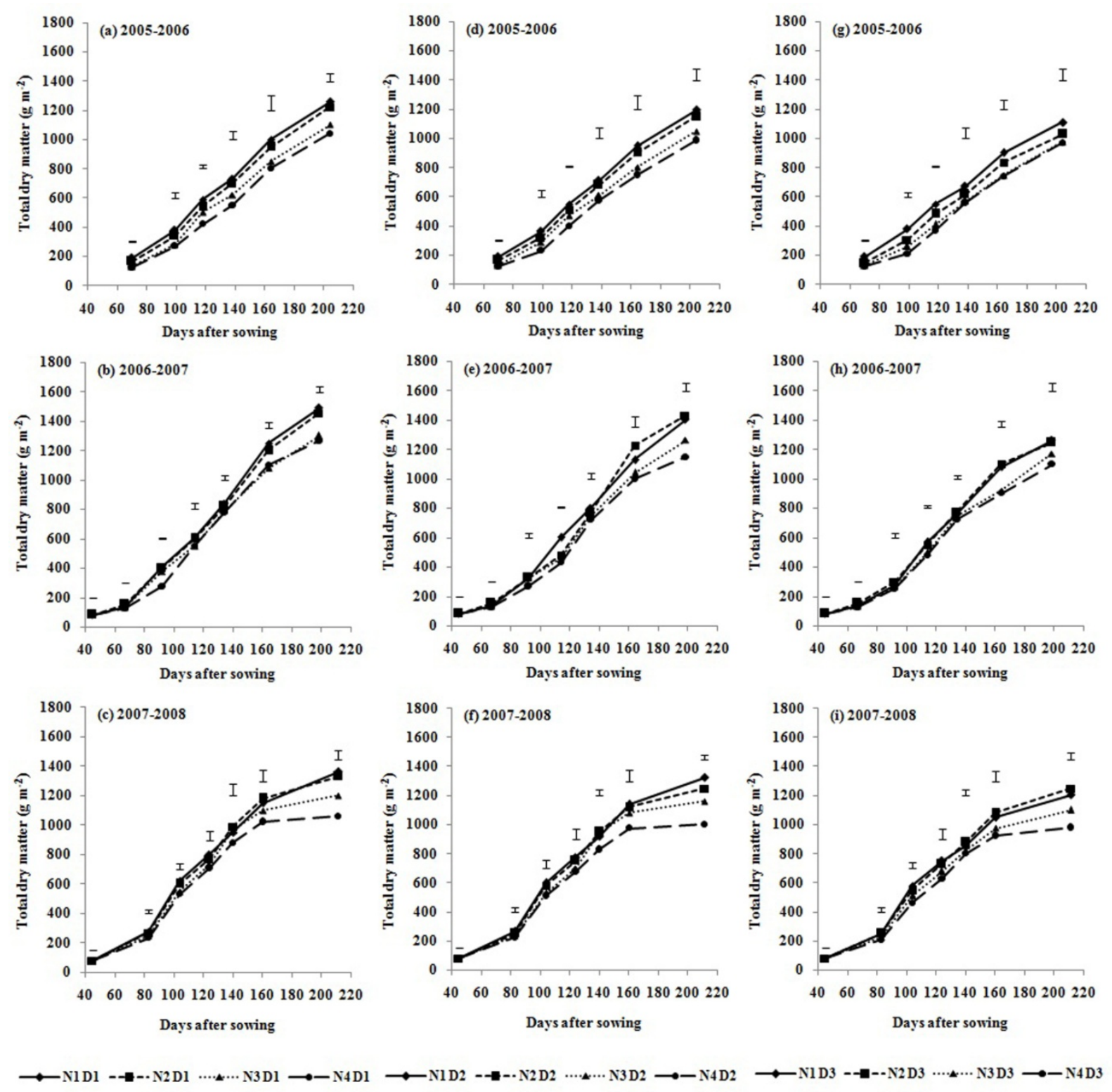

Figure 2. Total dry matter accumulation of wheat during the three experiments 2006; 2007 and 2008 at four nitrogen rates (N1, N2, N3 and N4) under irrigation doses D1 (a, b and c); D2 (d, e and f) and D3 (g, h and i).

The vertical bars represent the least significant difference at $5 \%$ (LSD)

\subsection{Radiation Interception}

The cumulative radiation interception of wheat (PARabs) at the various nitrogen rates (N1, N2, N3 and N4) and under the three tested irrigations rates (D1, D2 and D3) are shown in Figure 3.

Data analysis showed that at harvesting, the PARabs was significantly $(\mathrm{P}<0.01)$ affected by irrigation regimes (D1, D2 and D3) in 2006, 2007 and 2008 growing seasons [Figure. 3 and Table 4]. The irrigation regime D1 has increased respectively (PARabs) in the treatment $\mathrm{N} 1$ about (13.6; 16.4 and $67.3 \mathrm{MJ} \mathrm{m}^{-2}$ ) and (42.8; 93 and $99 \mathrm{MJ}$ $\mathrm{m}^{-2}$ ) relative to in D2 and D3. Similarly, the D1 enhanced PARabs in the treatment N2 compared to D2 and D3 respectively, $\left(23.4 ; 8.1\right.$ and $\left.63.6 \mathrm{MJ} \mathrm{m}^{-2}\right)$ and $\left(55.4 ; 35.1\right.$ and $\left.81.1 \mathrm{MJ} \mathrm{m}^{-2}\right)$. Likewise for the treatment N4, D1 increased respectively PARabs about (3.3; 4.2 and $4.3 \%)$ and (5.4; 7.2 and 9.1\%) compared to D2 and D3.

For the three experiments, results revealed that the cumulative PARabs was significantly $(\mathrm{P}<0.001)$ influenced by nitrogen rates. Therefore, the application of nitrogen in N1 and N2 rates increased the radiation interception compared to N3 and N4 rate. In fact, in the irrigation regime D1, the treatment N1 has increased respectively 
PARabs (from 4.3 to $7.9 \%$ and from 4.1 to $11.7 \%$ ) compared to the treatment N3 and N4. Also, in the second regime D2, the treatment $\mathrm{N} 1$ has enhanced PARabs (from 1.9 to $9 \%$ and from 5.2 to $13.3 \%$ ) next to the treatment N3 and N4. Similarly, in the third regime D3, the treatment N1 has raised respectively PARabs (from 2.2 to $10.6 \%$ and from 2.2 to $12.3 \%$ ) compared to N3 and N4.

Variance analysis showed that there was no significant effect $(\mathrm{P}<0.05)$ of interaction between irrigation regime and nitrogen rates on the cumulative $\mathrm{PAR}_{\mathrm{abs}}$. However, this combined effect has a consequence on the cumulative PARabs during the three experiments. So, at harvesting, the cumulative PAR $_{a b s}$ in treatment D1N1 was respectively equal to $\left(920.2 ; 1041.5\right.$ and $\left.1031.3 \mathrm{MJ} \mathrm{m}^{-2}\right)$ and it was respectively equivalent to $(769.3 ; 927.7$ and 867.7 $\mathrm{MJ} \mathrm{m}^{-2}$ ) for wheat in treatment D3N4.
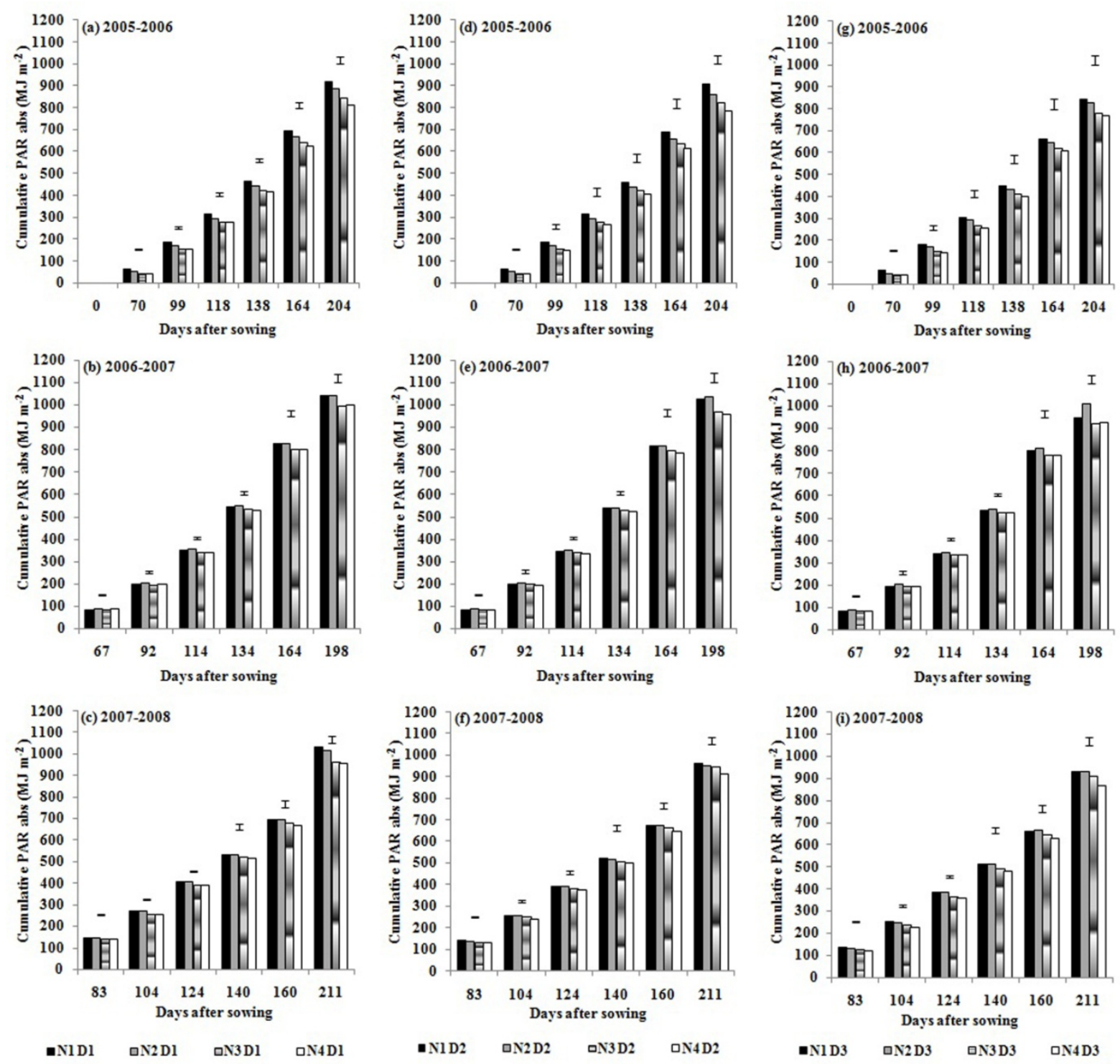

Figure 3. Time course of estimated cumulative light interception of wheat (PAR abs) in D1, D2 and D3 during the LAI measurement periods from 2006 to 2008 . The vertical bars represent the least significant difference at 5\% (LSD) 
Table 4. Cumulative light interception of wheat (PAR abs) at various nitrogen rates (N1, N2, N3 and N4) and under three irrigation doses (D1, D2 and D3) during the three campaigns (2006, 2007 and 2008)

\begin{tabular}{|c|c|c|c|c|c|c|c|c|c|c|c|c|c|c|c|c|c|c|}
\hline \multirow[b]{2}{*}{ DAS } & \multicolumn{5}{|c|}{$2005-2006$} & \multirow[b]{2}{*}{204} & \multicolumn{6}{|c|}{ 2006-2007 } & \multicolumn{5}{|c|}{$2007-2008$} & \multirow[b]{2}{*}{211} \\
\hline & 70 & 99 & 118 & 138 & 164 & & 67 & 92 & 114 & 134 & 164 & 198 & 83 & 104 & 124 & 140 & 160 & \\
\hline DIN1 & $64.9 \mathrm{a}$ & $187.8 \mathrm{a}$ & $316.8 \mathrm{a}$ & $465.2 \mathrm{a}$ & $695.1 \mathrm{a}$ & $920.2 \mathrm{a}$ & $83.1 \mathrm{~b}$ & $200.6 \mathrm{a}$ & $350.7 \mathrm{ab}$ & $544.7 \mathrm{ab}$ & $825.0 \mathrm{a}$ & $1041.5 \mathrm{a}$ & $145.8 \mathrm{a}$ & $267.6 \mathrm{a}$ & $405.8 \mathrm{a}$ & $533.6 \mathrm{a}$ & $695.9 \mathrm{a}$ & $1031.3 \mathrm{a}$ \\
\hline DIN2 & $50.6 \mathrm{~b}$ & $169.9 \mathrm{bc}$ & $5.8 \mathrm{~b}$ & $442.2 \mathrm{abc}$ & $668.0 \mathrm{abcd}$ & $885.6 \mathrm{abc}$ & $86.4 \mathrm{a}$ & $205.8 \mathrm{a}$ & $355.4 \mathrm{a}$ & $549.0 \mathrm{a}$ & $828.1 \mathrm{a}$ & $1042.7 \mathrm{a}$ & $45.8 \mathrm{a}$ & $267.1 \mathrm{a}$ & $404.6 \mathrm{a}$ & $532.5 \mathrm{a}$ & 694.4 a & $1013.9 \mathrm{a}$ \\
\hline N3 & $45.9 \mathrm{c}$ & $157.9 \mathrm{~cd}$ & $1.1 \mathrm{c}$ & $425.4 \mathrm{bcd}$ & $643.6 \mathrm{bcdef}$ & $847.6 \mathrm{cde}$ & $3.0 \mathrm{~b}$ & $196.5 \mathrm{a}$ & $342.4 \mathrm{abcd}$ & $533.7 \mathrm{abc}$ & $804.2 \mathrm{abcd}$ & $997.4 \mathrm{~cd}$ & $140.8 \mathrm{ab}$ & $257.6 \mathrm{ab}$ & $393.1 \mathrm{ab}$ & $520.7 \mathrm{ab}$ & $679.0 \mathrm{ab}$ & $966.0 \mathrm{~b}$ \\
\hline N4 & $44.4 \mathrm{~cd}$ & $154.5 \mathrm{~cd}$ & $4.8 \mathrm{~cd}$ & $15.2 \mathrm{~cd}$ & $623.3 \mathrm{def}$ & $12.7 \mathrm{ef}$ & $85.8 \mathrm{a}$ & $195.8 \mathrm{a}$ & $338.2 \mathrm{bcd}$ & $529.4 \mathrm{abc}$ & 801.2 abcd & $999.4 \mathrm{~cd}$ & $137.3 \mathrm{abc}$ & $252.1 \mathrm{abc}$ & $386.9 \mathrm{ab}$ & $513.7 \mathrm{abc}$ & $669.8 \mathrm{ab}$ & $954.0 \mathrm{bc}$ \\
\hline N1 & $9 \mathrm{a}$ & $186.4 \mathrm{ab}$ & $2.8 \mathrm{a}$ & $9.9 \mathrm{ab}$ & $7.7 \mathrm{ab}$ & $6.6 \mathrm{ab}$ & $3.3 \mathrm{~b}$ & $7.6 \mathrm{a}$ & $345.9 \mathrm{abc}$ & $539.1 \mathrm{abc}$ & $\$ 15.6 \mathrm{ab}$ & $1025.1 \mathrm{abc}$ & $139.5 \mathrm{ab}$ & $258.3 \mathrm{ab}$ & $393.6 \mathrm{ab}$ & $519.5 \mathrm{abc}$ & $674.9 \mathrm{ab}$ & $964.0 \mathrm{~b}$ \\
\hline N2 & $5 \mathrm{~b}$ & $8.1 \mathrm{c}$ & $1 \mathrm{~b}$ & $.4 \mathrm{abc}$ & 8.2 abcde & $2.2 \mathrm{~cd}$ & se & $3.5 \mathrm{a}$ & $7.0 \mathrm{abc}$ & $537.8 \mathrm{abc}$ & $815.5 \mathrm{abc}$ & $1034.6 \mathrm{ab}$ & $136.3 \mathrm{abc}$ & $253.2 \mathrm{abc}$ & $388.9 \mathrm{ab}$ & $515.5 \mathrm{abc}$ & $671.1 \mathrm{ab}$ & $950.3 \mathrm{bc}$ \\
\hline $2 \mathrm{~N} 3$ & $2 \mathrm{~cd}$ & $158.2 \mathrm{~cd}$ & $0.5 \mathrm{c}$ & $3 \mathrm{~cd}$ & & $3 \mathrm{def}$ & $84.2 \mathrm{ab}$ & $199.4 \mathrm{a}$ & $342.9 \mathrm{abcd}$ & $531.7 \mathrm{abc}$ & $795.1 \mathrm{abcd}$ & $971.8 \mathrm{de}$ & $136.1 \mathrm{abc}$ & $250.4 \mathrm{bc}$ & $383.7 \mathrm{abc}$ & $509.2 \mathrm{abc}$ & $663.5 \mathrm{abc}$ & $946.0 \mathrm{bc}$ \\
\hline $2 \mathrm{~N} 4$ & $7 \mathrm{de}$ & $150.3 \mathrm{~d}$ & $267.5 \mathrm{~d}$ & $407.4 \mathrm{~cd}$ & $612.0 \mathrm{ef}$ & & & $194.7 \mathrm{a}$ & $334.7 \mathrm{~cd}$ & $521.8 \mathrm{c}$ & $782.7 \mathrm{bcd}$ & $957.4 \mathrm{ef}$ & $130.4 \mathrm{bc}$ & $241.2 \mathrm{~cd}$ & $373.2 \mathrm{bc}$ & $497.5 \mathrm{bcd}$ & $647.5 \mathrm{bc}$ & $913.5 \mathrm{c}$ \\
\hline $3 \mathrm{N1}$ & & $188.3 \mathrm{a}$ & $315.7 \mathrm{a}$ & 461.8 a & $682.7 \mathrm{abc}$ & $877.4 \mathrm{bc}$ & $83.3 \mathrm{~b}$ & 195.1 a & 340.6 bcd & $531.7 \mathrm{abc}$ & 799.2 abcd & $948.5 \mathrm{efg}$ & $135.8 \mathrm{abc}$ & $252.4 \mathrm{abc}$ & $385.9 \mathrm{abc}$ & $510.3 \mathrm{abc}$ & $662.3 \mathrm{abc}$ & $932.3 \mathrm{bc}$ \\
\hline N2 & $\mathrm{b}$ & $168.1 \mathrm{c}$ & $290.8 \mathrm{~b}$ & $433.8 \mathrm{abcd}$ & 648.2 abcdef & $0.2 \mathrm{de}$ & $86.4 \mathrm{a}$ & $202.1 \mathrm{a}$ & $347.0 \mathrm{abc}$ & $537.9 \mathrm{abc}$ & 811.5 abcd & $1007.6 \mathrm{bc}$ & $132.6 \mathrm{abc}$ & $248.5 \mathrm{bc}$ & $383.7 \mathrm{abc}$ & 509.5 abc & $662.7 \mathrm{abc}$ & $932.8 \mathrm{bc}$ \\
\hline & & $2.4 \mathrm{~cd}$ & $270.8 \mathrm{~d}$ & $412.7 \mathrm{~cd}$ & $621.3 \mathrm{def}$ & $784.7 \mathrm{fg}$ & $84.2 \mathrm{ab}$ & $194.4 \mathrm{a}$ & $336.0 \mathrm{~cd}$ & $526.0 \mathrm{bc}$ & $781.1 \mathrm{~cd}$ & $921.8 \mathrm{~g}$ & $27.9 \mathrm{bc}$ & $237.3 \mathrm{~cd}$ & $368.3 \mathrm{bc}$ & $493.0 \mathrm{~cd}$ & $645.1 \mathrm{bc}$ & $912.4 \mathrm{c}$ \\
\hline & & $4.4 \mathrm{~d}$ & & & & & & $191.7 \mathrm{a}$ & $331.9 \mathrm{~d}$ & $521.0 \mathrm{c}$ & $777.9 \mathrm{~d}$ & $927.7 \mathrm{fg}$ & $122.0 \mathrm{c}$ & $227.3 \mathrm{~d}$ & $356.8 \mathrm{c}$ & $480.9 \mathrm{~d}$ & $628.4 \mathrm{c}$ & $867.7 \mathrm{~d}$ \\
\hline & & & & & & & & & & & 34.44 & & 15.39 & 16.01 & & 26.57 & 38.61 & \\
\hline D & $0.0472^{*}$ & $0.6069 \mathrm{NS}$ & $0.0027^{* *}$ & $0.5488 \mathrm{NS}$ & & & $9143 \mathrm{NS}$ & $.5186 \mathrm{NS}$ & $0.1152 \mathrm{NS}$ & $0.1245 \mathrm{NS}$ & $0.0417^{*}$ & $\overline{0.0001^{* * *}}$ & $0.0057 \mathrm{NS}$ & $0.0005 * * *$ & $0.0174^{*}$ & $0.0042^{* *}$ & $0.0081^{* *}$ & \\
\hline $\mathrm{s}$ & $0.0001^{* * *}$ & $0.0001^{* * * *}$ & $0.0001^{* * *}$ & $0.0004 * * *$ & $0.0007^{* * *}$ & & & & & & & & & $0.0031^{* *}$ & $0.0615 \mathrm{NS}$ & $0.0272^{*}$ & $0.0679 \mathrm{NS}$ & $1^{* * *}$ \\
\hline$D^{*} \mathrm{~N}$ & $0.6934 \mathrm{NS}$ & $0.9743 \mathrm{NS}$ & $0.1414 \mathrm{NS}$ & $0.9984 \mathrm{NS}$ & $1 \mathrm{NS}$ & $0.9855 \mathrm{NS}$ & $0.4674 \mathrm{NS}$ & $0.9977 \mathrm{NS}$ & $0.9808 \mathrm{NS}$ & $0.9911 \mathrm{NS}$ & $0.996 \mathrm{NS}$ & $0.1353 \mathrm{NS}$ & $0.9939 \mathrm{NS}$ & $0.9591 \mathrm{NS}$ & $0.9967 \mathrm{NS}$ & $0.9959 \mathrm{NS}$ & $0.9994 \mathrm{NS}$ & $0.361 \mathrm{NS}$ \\
\hline
\end{tabular}

DAS: Days After Sowings, LSD: Least Significant Difference, * significant difference at $5 \%$, ** significant difference at $0.01 \%$, *** significant difference at $0.001 \%$, NS no significant difference at $5 \%$.

\subsection{Radiation Use Efficiency}

The radiation use efficiency of wheat (RUE) in the three irrigation level (D1, D2 and D3) and during the three experiments $(2005 ; 2006$ and 2007) is given in Figure 4. RUE presents a high variability according to the treatments and years (Figure 4 and Table 5). The maximum of RUE $\left(1.59 \mathrm{~g} \mathrm{MJ}^{-1}\right)$ was recorded under treatment D2N1 in year 3. The lowest RUE value $\left(1.24 \mathrm{~g} \mathrm{MJ}^{-1}\right)$ was recorded in year 2 under treatment D3N4. Data analysis showed that RUE was significantly $(\mathrm{P}<0.001)$ affected by irrigation regimes (D1, D2 and D3) in 2006, 2007 and 2008 (Table 5).

In the end of maturity stage, the irrigation regime D1 has improved respectively RUE in the treatment N1 (from 3.4 to $6 \%$ ) and (from 6.8 to $8.7 \%$ ) relative to in D2 and D3. Similarly, the D1 enhanced respectively RUE in the treatment $\mathrm{N} 2$ (from 1.4 to $3.4 \%$ ) and (from 8.3 to $9.6 \%$ ) relative to in D2 and D3. Likewise for the treatment N4, D1 increased respectively RUE (from 1.5 to $6.7 \%$ ) and (from 2.3 to $8.1 \%$ ) relative to D2 and D3.

The RUE was the greatest in both N1 and N2 treatments and the least in the treatment N4 overall years and irrigation regimes (D1, D2 and D3) [Figure. 4 and Table 5]. ANOVA revealed that RUE was significantly (P < 0.001 ) influenced by $\mathrm{N}$ rates. Therefore, the two treatments $\mathrm{N} 1$ and $\mathrm{N} 2$ increased significantly RUE compared to $\mathrm{N} 3$ and N4. In fact, in the irrigation regime D1, the treatment N1 has improved respectively RUE from 2.6 to $8.7 \%$ and from 10 to $10.3 \%$ compared to $\mathrm{N} 3$ and N4. Also, in the second irrigation regime D2, the treatment N1 has enhanced RUE compared to N3 and N4, respectively from 5.7 to $6.4 \%$ and from 8.5 to $13.2 \%$. Similarly, in the third irrigation regime D3, the treatment N1 has improved respectively RUE from 5.3 to $6.6 \%$ and from 5.9 to $9.5 \%$ compared to $\mathrm{N} 3$ and $\mathrm{N} 4$.

Variance analysis showed that there was no significant effect $(\mathrm{P}<0.05)$ of interaction between irrigation regime and nitrogen rates on RUE. For the three experiments (2006, 2007 and 2008), the RUE in treatment D1N1 was respectively equal to $1.46 ; 1.5$ and $1.55 \mathrm{~g} \mathrm{MJ}^{-1}$ and it was respectively equivalent to $1.28 ; 1.24$ and $1.38 \mathrm{~g} \mathrm{MJ}^{-1}$ in treatment D3N4. 
Table 5. Radiation use efficiency of wheat (RUE) at the four nitrogen rates (N1, N2, N3 and N4) and under the three irrigation doses (D1, D2 and D3) during the three growing season (2006, 2007 and 2008)

\begin{tabular}{lcccccc}
\hline & \multicolumn{2}{c}{ 2005-2006 } & \multicolumn{2}{c}{ 2006-2007 } & \multicolumn{2}{c}{ 2007-2008 } \\
\cline { 2 - 7 } & RUE & $\mathbf{R}^{2}$ & RUE & $\mathbf{R}^{2}$ & RUE & $\mathbf{R}^{2}$ \\
\hline D1N1 & $1.46 \mathrm{a}$ & 0.96 & $1.5 \mathrm{a}$ & 0.98 & $1.55 \mathrm{abc}$ & 0.91 \\
D1N2 & $1.46 \mathrm{a}$ & 0.97 & $1.45 \mathrm{~b}$ & 0.98 & $1.56 \mathrm{abc}$ & 0.91 \\
D1N3 & $1.36 \mathrm{bc}$ & 0.97 & $1.37 \mathrm{~cd}$ & 0.98 & $1.51 \mathrm{abc}$ & 0.9 \\
D1N4 & $1.31 \mathrm{~cd}$ & 0.98 & $1.35 \mathrm{de}$ & 0.98 & $1.39 \mathrm{~d}$ & 0.86 \\
D2N1 & $1.41 \mathrm{ab}$ & 0.96 & $1.41 \mathrm{bc}$ & 0.99 & $1.59 \mathrm{a}$ & 0.93 \\
D2N2 & $1.41 \mathrm{ab}$ & 0.96 & $1.43 \mathrm{~b}$ & 0.99 & $1.57 \mathrm{ab}$ & 0.91 \\
D2N3 & $1.32 \mathrm{~cd}$ & 0.97 & $1.32 \mathrm{ef}$ & 0.99 & $1.50 \mathrm{c}$ & 0.90 \\
D2N4 & $1.29 \mathrm{~d}$ & 0.98 & $1.26 \mathrm{~g}$ & 0.99 & $1.38 \mathrm{~d}$ & 0.86 \\
D3N1 & $1.36 \mathrm{bc}$ & 0.95 & $1.37 \mathrm{~cd}$ & 0.99 & $1.51 \mathrm{bc}$ & 0.91 \\
D3N2 & $1.32 \mathrm{~cd}$ & 0.96 & $1.33 \mathrm{de}$ & 0.98 & $1.54 \mathrm{abc}$ & 0.93 \\
D3N3 & $1.28 \mathrm{~d}$ & 0.97 & $1.28 \mathrm{fg}$ & 0.98 & $1.43 \mathrm{~d}$ & 0.90 \\
D3N4 & $1.28 \mathrm{~d}$ & 0.98 & $1.24 \mathrm{~g}$ & 0.98 & $1.38 \mathrm{~d}$ & 0.89 \\
\hline LSD (5\%) & 0.067 & \multicolumn{7}{c}{0.045} & 0.066 & \\
\hline $\mathbf{D}$ & $0.0004^{* * *}$ & $0.0001^{* * *}$ & & $0.0053^{* *}$ & \\
$\mathbf{N}$ & $0.0001^{* * *}$ & $0.0001^{* * *}$ & & $0.0001^{* * *}$ & \\
D*N & $0.5574 \mathrm{NS}$ & $0.1191 \mathrm{NS}$ & $0.2728 \mathrm{NS}$ & \\
\hline
\end{tabular}

DAS: Days After Sowings, LSD: Least Significant Difference, * significant difference at $5 \%$, ** significant difference at $0.01 \%$, *** significant difference at $0.001 \%$, NS no significant difference at $5 \%$.
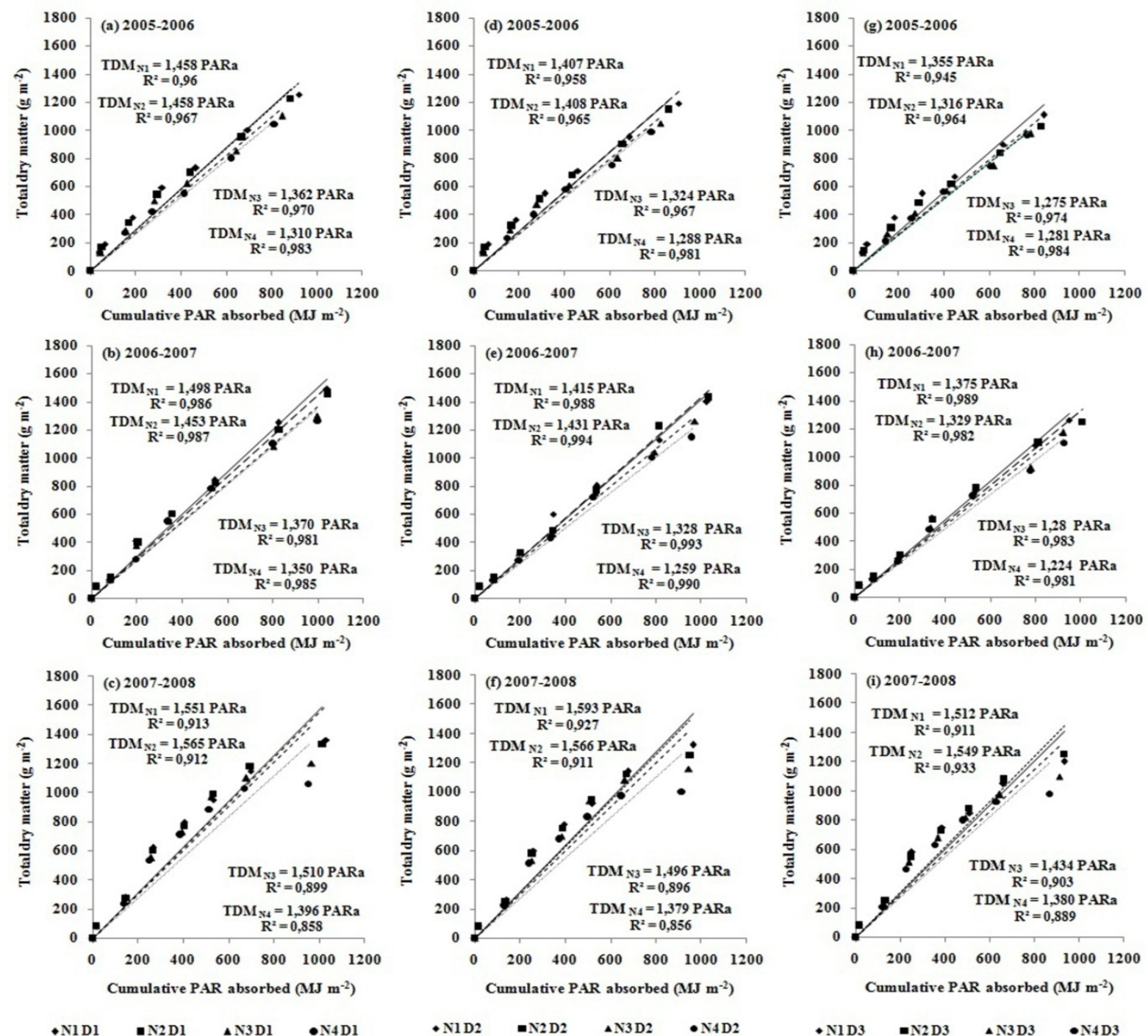

$\bullet \mathrm{N} 1 \mathrm{D} 1 \quad \bullet \mathrm{N} 2 \mathrm{D} 1 \quad \triangle \mathrm{N} 3 \mathrm{Dl} \quad \bullet \mathrm{N} 4 \mathrm{Dl}$

$\bullet \mathrm{N} 1 \mathrm{D} 2 \quad-\mathrm{N} 2 \mathrm{D} 2 \quad \Delta \mathrm{N} 3 \mathrm{D} 2 \quad \bullet \mathrm{N} 4 \mathrm{D} 2$

$\bullet \mathrm{N} 1 \mathrm{D} 3 \quad-\mathrm{N} 2 \mathrm{D} 3 \quad \triangle \mathrm{N} 3 \mathrm{D} 3 \quad \bullet \mathrm{N} 4 \mathrm{D} 3$

Figure 4. Radiation use efficiency of wheat during the three experiments 2006; 2007 and 2008 in D1 (a, b and c); in D2 (d, e and f) and in D3 (g, h and i) 


\subsection{Discussion}

The combinated effects of the three irrigation amount (D1, D2 and D3) and the four nitrogen rates (N1, N2, N3 and $\mathrm{N} 4)$ on the leaf area index (LAI), total dry matter production (TDM), radiation interception (PARabs) and radiation use efficiency (RUE) were investigated. As shown by the results (Table 2), LAI was decreased by deficit irrigation and low nitrogen rates (Figure 1). These results were in agreement with those of Khaliq et al. (1999). The latter authors observed that an increase in nitrogen content of soil affects all growth stages of wheat. Salvagiotti and Miralles (2008) found that an increase in nitrogen concentration at anthesis can result in an increase of LAI by as much as $62 \%$ and IPAR by up to $20 \%$. As well, numerous researchers affirmed that under nitrogen shortage the leaf area expansion decreases and senescence increases (Vos \& Biemond, 1992; Massignam et al., 2011). Likewise, these results are consistent with those of Collinson et al. (1999). These authors reported that the water deficit reduces the solar radiation interception due to rolling up the leaves and they observed that the number and size of leaves may be reduced or the total leaf area may decrease, if the water deficit is prolonged. In fact, the optimum nitrogen (N2), high doses (N1) and irrigation amount (D1) had greater contributions to leaf expansion as a consequence of higher growth rate of leaf area. Furthermore, we observed that irrigation amount and nitrogen application rates affect the TDM accumulation. This effect could be due to water availability in (D2 and D3) and to the low nitrogen accessibility for plant under (N3 and N4) treatments, which in result in the aboveground growth restriction. Definitely, the highest amount of TDM was obtained under the D1N1 condition (Table 3). With reduced nitrogen (N3 and N4) and irrigation application rates (D2 and D3), TDM decreased and the lowest values was observed under D3N4 condition. These findings are in line with those of Gan et al. (2008), Ali et al. (2009), Hamzei (2011) and Hamzei et al. (2012). They observed that increasing nitrogen levels increase the biological yield of the crop. Ezzat-Ahmadi (2002) showed that the level of $160 \mathrm{~kg}$ of nitrogen produced the highest yield. MacDonald (2002) examined different nitrogen levels on the yield of different wheat cultivars and he observed that dry matter at anthesis significantly increased at increased nitrogen. Tewolde and Fernandez (1997) confirmed that the nitrogen deficiency reduces vegetative and reproductive growth with a final impact on the yield due to leaf senescence. Nevertheless, Nielsen et al. (2002) reported that the wheat grain yield, photosynthesis, and total dry matter accumulation decreased with over-fertilization of nitrogen. Therefore, determination of the appropriate amount of nitrogen for dry land wheat is important, so that the growers can optimize yields and improve their grain quality without over fertilizing with $\mathrm{N}$ that might increase $\mathrm{N}$ leaching potential (Halvorson et al., 2004). As analyses indicated, irrigation regimes and nitrogen rates had significant effects on cumulative radiation interception (Table 4). In fact, the highest amount of cumulative PARabs was obtained under the D1NI condition. With reduced $\mathrm{N}$ application rates and irrigation doses, PARabs also decreased and the lowest values were observed under D3N4 condition. According to Caviglia and Sadras (2001), the LAI were reduced in crops grown under nitrogen deficiency. Also, Dreccer et al. (2000) observed that low nitrogen conditions affected wheat growth via reduction of the intercepted PAR. The reduction should be on the leaf area dynamics to limit IPAR in arid environments (O'Connell et al., 2004; Miranzadeh et al., 2011). As shown by data (Table 5), RUE was affected and decreased under deficit irrigation and low nitrogen conditions (figure 4). The RUE varied from $1.55 \mathrm{~g} \mathrm{MJ}^{-1}$ (D1N1) to $1.24 \mathrm{~g} \mathrm{MJ}^{-1}$ (D3N4). These findings are in line with those Gregory et al. (1992); Yunusa et al (1993); Latiri-Souki et al. (1998). They found that for wheat in semi-arid conditions and at different irrigation and nitrogen levels, the conversion efficiency of the incident PAR varies between $0.9 \mathrm{~g} \mathrm{MJ}^{-1}$ for treatments without irrigation and without nitrogen and $1.5 \mathrm{~g} \mathrm{MJ}^{-1}$ for treatments with irrigation and nitrogen. However, the conversion efficiency calculated for PAR intercepted, the values are higher and vary between $1.4 \mathrm{~g} \mathrm{MJ}^{-1}$ and $2.9 \mathrm{~g} \mathrm{MJ}^{-1}$ between treatments. Furthermore, these results were in agreement with those of Caviglia and Sadras (2001) and Muurinen and Peltonen-Sainio (2006). They affirmed that the RUE of wheat was reduced when nitrogen was limited. Similarly, Fletcher et al. (2013) observed that under nitrogen deficit the RUE with $200 \mathrm{~kg} \mathrm{~N}^{-1}$ was 1.66 $\mathrm{g} \mathrm{MJ}^{-1}$ PAR, which fell by $22 \%$ to $1.30 \mathrm{~g} \mathrm{MJ}^{-1}$ PAR when no $\mathrm{N}$-fertilizer was applied. Wilson and Jamieson (1985) observed in arid environments, that water stress tends to reduce RUE progressively by preventing utilization of photosynthates for growth as lower IPAR occurs from reduced LAI. Likewise, the reductions in RUE due to water deficits have been reported by Hughes and Keatinge (1983) in grain legumes. In this study, TDM accumulation was positively related to interception of PAR (Figure. 4), which is a finding in line with the results reported by other researchers (Li et al., 2009; Miranzadeh et al., 2011, Rezig et al., 2013a, 2013b). Miralles and Slafer (1997) indicated that post-anthesis RUE appeared to be closely and positively associated with the number of grains set per unit biomass at anthesis in winter wheat. Whitfield and Smith (1989), Chen et al. (2003), and Li et al. (2008) showed that crop yield was positively related to RUE in winter wheat. Equally, different crops have been found to be closely correlated with cumulative radiation intercepted by their foliage e.g. Sulla (Rezig et al., 2013b), green been (Rezig et al., 2010, 2013a) and potato (Rezig et al., 2010, 2013a, 2013b). 


\section{Conclusion}

LAI, TDM, PARabs and RUE were affected by different irrigation regimes and nitrogen rates. Nitrogen application ( $11=150 \mathrm{~kg} \mathrm{~N} / \mathrm{ha}$ and N2 $=100 \mathrm{~kg} \mathrm{~N} / \mathrm{ha})$ and full irrigation could accelerate leaf area development and help to intercept more radiation for dry matter production. In fact, water deficit in D3 and nitrogen deficiency $(\mathrm{N} 3=50 \mathrm{~kg} \mathrm{~N} / \mathrm{ha}$ and N4 $=0 \mathrm{~kg} \mathrm{~N} / \mathrm{ha}$ ) treatments caused a high reduction of TDM followed by a greater LAI sensitivity. Higher RUE $\left(1.56 \mathrm{~g} \mathrm{MJ}^{-1}\right)$ was recorded under full irrigation (D1) combined with optimum $\mathrm{N}$ application $(\mathrm{N} 2=100 \mathrm{~kg} \mathrm{~N} / \mathrm{ha})$ and the lowest values $\left(1.24 \mathrm{~g} \mathrm{MJ}^{-1}\right)$ was observed under deficit irrigation and nitrogen deficiency (D3N4). So, D1N1 and D1N2 treatments can be recommended for durum wheat .cv. Karim under field conditions in semi arid zone of Tunisia in order to improve RUE and maximize the yield. Further studies on the influence of nitrogen $\times$ irrigation interactions on RUE are needed to enhance water use efficiency and yield potential of durum wheat .cv. Karim under different conditions.

\section{References}

Ali, M. A., Aslam, M., Hammad, H. M., Abbas, G., Akram, M., \& Ali, Z. (2009). Effect of nitrogen application timings on wheat yield under thal environment. J. Agric. Res, 47(1), 31-35.

Allen, R. G., Pereira, L. S., Raes, D., \& Smith, M. (1998). Crop evapotranspiration: guidelines for computing crop water requirements. FAO Irrigation and Drainage Paper, 56 (p. 300).

Aggarwal, P. K., \& Karla, N. (1994). Analyzing the limitations set by climatic factors, genotype, and water and nitrogen availability on productivity of water. II. Climatically potential yields and management strategies. Field Crops Res, 38, 93-103. http://dx.doi.org/10.1016/0378-4290(94)90002-7

Belder, P., Bouman, B. A. M., Cabangon, R., Lu, G., Quilang, E. J. P., Li, Y., ... Tuong, T. P. (2004). Effect of water-saving irrigation on rice yield and water use in typical lowland conditions in Asia. Agric. Water Manage, 65, 193-210. http://dx.doi.org/10.1016/j.agwat.2003.09.002

Bouman, B. A. M., Humphreys, E., Tuong, T. P., \& Barker, R. (2006). Rice and water. Adv. Agron, 92, 187-237. http://dx.doi.org/10.1016/S0065-2113(04)92004-4

Campbell, C. A., Zentner, R. P., Selles, F., McConkey, B. G., \& Dyck, F. B. (1993a). Nitrogen management for spring wheat grown annually on zero-tillage: yields and nitrogen use efficiency. Agron. J, 85, 107-114. http://dx.doi.org/10.2134/agronj1993.00021962008500010021x

Campbell, C. A., Selles, F., Zentner, R. P., \& McConkey, B. G. (1993b). Available water and nitrogen effects on yield components and grain nitrogen of zero-till spring wheat. Agron. J, 85, 114-120. http://dx.doi.org/10.2134/agronj1993.00021962008500010021x

Caviglia, O. P., \& Sadras, V. (2001). Effect of Nitrogen Supply on Crop Conductance, Water-and Radiation-use Efficiency of Wheat. Field Crops Res., 69, 259-266. http://dx.doi.org/10.1016/S0378-4290(00)00149-0

Chen, Y. H., Yu, S. L., \& Yu, Z. W. (2003). Relationship between Amount or Distribution of PAR Interception and Grain Output of Wheat Communities. Acta Agron. Sin., 29, 730-734.

Collino, D. J., Dardanelli, J. L., Sereno, R., \& Racca, R. W. (2001). Physiological responses of argentine peanut varieties to water stress. Light interception, radiation use efficiency and partitioning of assimilates. Field Crops Res, 70, 177-184. http://dx.doi.org/10.1016/S0378-4290(01)00137-X

Collinson, S. T., Berchie, J., \& Azam-Ali, S. N. (1999). The effect of soil moisture on light interception and the conversion coefficient for three landraces of Bambara groundnut (Vigna subterranea). Journal of Agricultural Science, 133, 151-157. http://dx.doi.org/10.1017/S0021859699006875

Dreccer, M. F., Schapendonk, A. H. C. M., Slafer, G. A., \& Rabbinge, R. (2000). Comparative Response of Wheat and Oilseed Rape to Nitrogen Supply: Absorption and Utilization Efficiency of Radiation and Nitrogen during the Reproductive Stages Determining Yield. Plant Soil, 220, 189-205. http://dx.doi.org/10.1023/A:1004757124939

Ezzat-Ahmadi A. (2002). Effect of nitrogen fertilizer on photosynthesis and ribulose 1,5-diphosphate carboxylase activity in spring wheat in the field. Journal of Exp. Bot, 26, 43-51.

Fletcher, A. L., Johnstone, P., Chakwizira, E., \& Browen, H. E., (2013). Radiation capture and radiation use efficiency in response to $\mathrm{N}$ supply for crop species with contrasting canopies. Field Crops Research, 150, 126-134. http://dx.doi.org/10.1016/j.fcr.2013.06.014 
Gan, Y., Malhi, S. S., Brandt, S., Katepa-Mupondwad, F., \& Stevenson, C., (2008). Nitrogen use efficiency and nitrogen uptake of juncea canola under diverse environments. Agron. J., 100, 285-295. http://dx.doi.org/10.2134/agrojn12007.0229

Gregory, P. J., Tenant, D., \& Belford, R. K. (1992). Root and shoot growth and water and light use efficiency of barley and wheat crops grown on a shallow Duplex soil in a Mediterranean type environment. Aust. J. Agric. Res, 43, 555-573. http://dx.doi.org/10.1071/AR9920555

Grindlay, D. J. C. (1997). Towards an explanation of crop nitrogen demand based on the optimization of leaf nitrogen per unit leaf area. J. Agric. Sci., 128, 377-396. http://dx.doi.org/10.1017/S0021859697004310

Halvorson, A. D., Nielsen, D. C., \& Reule, C. A. (2004). Nitrogen Fertilization and Rotation Effects on No-till Dryland Wheat Production. Agron. J., 96, 1196-1201. http://dx.doi.org/10.2134/agronj2004.1196

Hamzei, J. (2011). Seed, oil, and protein yields of canola under combinations of irrigation and nitrogen application. Agron. J., 103, 1152-1158. http://dx.doi.org/10.2134/agronj2011.0018

Hamzei J., \& Soltani, J. (2012). Deficit irrigation of rapeseed for water-saving: Effects on biomass accumulation, light interception and radiation use efficiency under different $\mathrm{N}$ rates. Agriculture, Ecosystems and Environment, 155, 153-160. http://dx.doi.org/10.1016/j.agee.2012.04.003

Howard, D. D., Gwathmey, C. O., Essington, M. E., Roberts, R. K., \& Mullen, M. D. (2001). Nitrogen fertilization of no-till cotton on loess-derived soils. Agron. J., 93, 157-163. http://dx.doi.org/10.2134/agronj2001.931157x

Hughes, G., \& Keatinge, J. D. H. (1983). Solar radiation interception, dry matter production and yield in pigeon

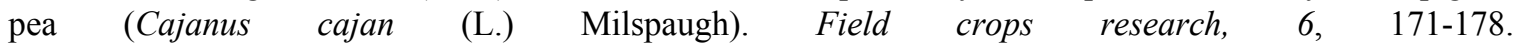
http://dx.doi.org/10.1016/0378-4290(83)90058-8

Jabloun, M., \& A. Sahli., (2008). Evaluation of FAO-56 methodology for estimating reference evapotranspiration using limited climatic data Application to Tunisia. Agricultural water management, 95, 707-715. http://dx.doi.org/10.1016/j.agwat.2008.01.009

Jamieson, P. D., Brooking, I. R., Porter, J. R., \& Wilson, D. R. (1995). Prediction of leaf appearance in wheat: a question of temperature. Field Crops Research, 41(1), 35-44. http://dx.doi.org/10.1016/0378-4290(94)00102-I

Khaliq A, Iqbal M., \& Basra, S. M. A. (1999). Optimization of seeding density and nitrogen application in wheat cv. Inqlab-91 under Faisalabad conditions. Int. J. Agric. Biol, 1(4), 241-243.

Katsura, K., Maeda, S., Horie, T., \& Shiraiwa, T. (2007). Analysis of yield attributes and crop physiological traits of Liangyoupeijiu, a hybrid rice recently bred in China. Field Crops Res, 103, 170-177. http://dx.doi.org/10.1016/j.fcr.2007.06.001

Katsura, K., Maeda, S., Lubis, I., Horie, T., Cao, W., \& Shiraiwa, T. (2008). The high yield of irrigated rice in Yunnan China: a cross-location analysis. Field Crops Res, 107, 1-11. http://dx.doi.org/10.1016/j.fcr.2007.12.007

Latiri-Souki, K. (1994). Analysis of the effects of water and nitrogen supply on the yield and growth of durum wheat unser semi-arid conditions in Tunisia. $\mathrm{PhD}$ Thesis, University of Reading. U K.

Latiri-Souki, Nortcliff, K, S., \& Lawlor, D. W. (1998). Nitrogen fertilizer can increase dry matter, grain production and radiation and water use efficiencies for durum wheat under semi-arid conditions. European Journal of Agronomy, 9, 21-34. http://dx.doi.org/10.1016/S1161-0301(98)00022-7

Li, Q. Q., Chen, Y. H., Liu, M. Y., Zhou, X. B., Yu, S. L., \& Dong, B. D. (2008). Effects of irrigation and planting patterns on radiation use efficiency and yield of winter wheat in North China. Agric. Water Management., 95, 469-476. http://dx.doi.org/10.1016/j.agwat.2007.11.010

Li, Q., Liu, M., Zhang, J., Dong, B., \& Bai, Q. (2009). Biomass accumulation and radiation use efficiency of winter wheat under deficit irrigation regimes. Plant Soil Environ, 55(2), 85-91. http://81.0.228.28/publicFiles/04099.pdf

Little, T. M., \& Hills, F. J. (1978). Agricultural Experimentation: Design and Analysis (p. 350). John Wiley \& Sons. New York-USA. 
MacDonald, G. K. (2002). Effects of nitrogenous fertilizer on the growth, grain yield and grain protein concentration of wheat. Aust. Journal of Agronomy Research, 43(5), 949-967. http://dx.doi.org/10.1071/AR9920949

Manrique, L. A., Kiniry, J. R., Hodges, T., \& Axness, D. S. (1991). Dry matter production and radiation interception of potato. Crop Sci., 31, 1044-1049. http://dx.doi.org/10.2135/cropsci1991.0011183X003100040040x

Massignam, A. M., Chapman, S. C., Hammer, G. L., \& Fukai, S. (2011). Effects of nitrogen supply on canopy development of maize and sunflower. Crop Pasture Sci, 62, 1045-1055. http://dx.doi.org/10.1071/CP11165

Miralles, D. J., \& Slafer, G. A. (1997). Radiation Interception and Radiation Use Efficiency of Near-isogenic Wheat Lines with Different Height. Euphytica, 97, 201-208. http://dx.doi.org/10.1023/A:1003061706059

Miranzadeh, H., Emam, Y., Seyyed, H., \& Zare, S. (2011). Productivity and radition use effieceny of four dry land wheat cultivars under different levels of nitrogen and chlormequat chloride. J. Agri. Sci. Tech, 13, 339-351.

Monteith, J. L., \& Elston, J. (1983). Performance and productivity of foliage in the field. In Growth and functioning of leaves: proceedings of a symposium held prior to the $13^{\text {th }}$ International Botanical Congress at the University of Sydney, 18-20 August 1981/edited by JE Dale and FL Milthorpe.

Monteith, J. L., \& Unsworth, M. (1990). Principles of Environmental Physics (2nd ed.). Edward. Arnold, London.

Muurinen S., \& Peltonen-Sainio P. (2006). Radiation-use efficiency of modern and old spring cereal cultivars and its response to nitrogen in northern growing conditions. Field Crops Research, 96, 363-373. http://dx.doi.org/10.1016/j.fcr.2005.08.009

Nielsen, D. C., Vigil, M. F., Anderson, R. L., Bowman, R. A., Benjamin, J. G., \& Halvorson, A. D. (2002). Cropping System Influence on Planting Water Content and Yield of Winter Wheat. Agron. J., 94, 962-967. http://dx.doi.org/10.2134/agronj2002.0962

O'Connell, M. G., O'Leary, G. J., Whitfield, D. M., \& Connor, D. J. (2004). Interception of Photosynthetically Active Radiation and Radiation-use Efficiency of Wheat, Field Pea and Mustard in a Semi-arid Environment. Field Crops Res., 85, 111-124. http://dx.doi.org/10.1016/S0378-4290(03)00156-4

Oweis, T., Pala, M., \& Ryan, J. (1999). Management alternatives for improved durum wheat production under supplemental irrigation in Syria. Eur. J. Agron, 11, 225-266. http://dx.doi.org/10.1016/S1161-0301(99)00036-2

Oweis, T., \& Hachum, A. Y. (2003). Improving Water Productivity in the Dry Areas of West Asia and North Africa. In W. Kijne, R. Barker \& D. Molden (Eds.) In CAB International 2003 - Water Productivity in Agriculture: Limits and Opportunities for Improvement, pp. 179-198.

Pala, M., Matar, A., \& Mazid, A. (1996). Assessment of the effects of environmental factors on the response of fertilizer in on-farm trails in a Mediterranean type environment. Exp. Agric, 32, 339-349. http://dx.doi.org/10.1017/S0014479700026272

Rezig, M., Sahli, A., Ben Jeddi., F., \& Harbaoui, Y. (2010). Adopting Intercropping System for Potatoes as Practice on Drought Mitigation under Tunisian Condition. Option Mediterranean, 95, 329-334.

Rezig, M., Sahli, A., Hachicha, M., Ben jeddi., F., \& Harbaoui, Y. (2013a). Potato (Solanum tuberosum L.) and Bean (Phaseolus vulgaris L.) In Sole Intercropping: Effects on Light Interception and Radiation Use Efficiency. Journal of Agricultural Science, 5(9), 65-77. http://dx.doi.org/10.5539/jas.v5n9p65

Rezig, M., Sahli, A., Ben Jeddi., F., \& Hachicha, M. (2013b). Light Interception and Radiation Use Efficiency from a field of Potato (Solanum tuberosum L.) and Sulla (Hedysarum coronarium L.) Intercropping in Tunisia. Asian Journal of Crop Science, 5(4), 378-392. http://dx.doi.org/10.3923/ajcs.2013.378.392

Salvagiotti, F., \& Miralles, D. J. (2008). Radiation Interception, Biomass Production and Grain Yield as Affected by the Interaction of Nitrogen and Sulfur Fertilization in Wheat. Europ. J. Agron., 28, 282-290. http://dx.doi.org/10.1016/j.eja.2007.08.002

Sakiss, N., Ennabli, N., Slimani, M. S., \& Baccour, H. (1994). La pluviométrie en Tunisie a-t-elle changée depuis 2000 ans: Recherche de tendances et de cycles dans les séries pluviométriques. Institut National de la Météorologie (INM), Institut National Agronomique de Tunis (INAT) \& Agence Nationale de Protection de l'Environnement (ANPE). p. 283. Tunis.

SAS Institute. (1985). SAS user's guide: Statistics. Version 6.0. SAS Inst. Inc., Cary, NC. USA. 
Sinclair, T. R., \& Muchow, R. C. (1999). Radiation use efficiency. Adv. Agron, 65, 215-265. http://dx.doi.org/10.1016/S0065-2113(08)60914-1

Sinclair, T. R., \& Weiss, A. (2010). Principles of ecology in plant production. CAB International, Wallingford, UK. http://dx.doi.org/10.1017/S0014479711000688

Tewolde, H., \& Fernandez, C. J. (1997). Vegetative and reproductive dry weight inhibition in nitrogen and phosphorus-deficient Pima cotton. J. Plant Nutr, 20, 219-232. http://dx.doi.org/10.1080/01904169709365245

Vos, J., \& Biemond, H., (1992). Effects of nitrogen on the development and growth of the potato plant. 1. Leaf appearance, expansion growth, life spans of leaves and stem branching. Ann. Bot, 70, 27-35. http://aob.oxfordjournals.org/content/70/1/27.full.pdf

Whitfield, D. M., \& Smith, C. J. (1989). Effect of irrigation and nitrogen on growth, light interception and efficiency of light conversion in wheat. Field Crops Res., 20, 279-295. http://dx.doi.org/10.1016/0378-4290(89)90071-3

Williams, J. H., \& Boote, K. J. (1995). Physiology and modelling predicting the unpredictable legume. In H. E. Pattee \& H. T. Stalker (Eds.), Advances in Peanut Science. Am. Peanut Res. Educ. Soc., Stillwater, OK.

Wilson, D. R., \& Jamieson, P. D. (1985). Models of Growth and Water Use of Wheat in New Zealand. In W. Day \& R. K. Atkin (Eds.). Wheat Growth and Modeling (pp. 211-216). London: Plenum Press. http://dx.doi.org/10.1007/978-1-4899-3665-3_21

Xu, Z. Z., Yu, Z. W., \& Wang, D. (2006). Nitrogen translocation in wheat plants under soil water deficit. Plant Soil, 280, 291-303. http://dx.doi.org/10.1007/s11104-005-3276-2

Yunusa, I. A. M., Siddique, K. H. M., Belford, R. K., \& Karimi, M. M. (1993). Effect of canopy structure on efficiency of radiation interception and use in spring wheat cultivars during preanthesis period in a Mediterranean-type environment. Field Crops Research, 35, 113-122. http://dx.doi.org/10.1016/0378-4290(93)90144-C

\section{Copyrights}

Copyright for this article is retained by the author(s), with first publication rights granted to the journal.

This is an open-access article distributed under the terms and conditions of the Creative Commons Attribution license (http://creativecommons.org/licenses/by/3.0/). 